\title{
ON ALGEBRAIC INTEGRABILITY OF GELFAND-ZEITLIN FIELDS
}

\author{
MARK COLARUSSO AND SAM EVENS
}

\begin{abstract}
We generalize a result of Kostant and Wallach concerning the algebraic integrability of the Gelfand-Zeitlin vector fields to the full set of strongly regular elements in $\mathfrak{g l}(n, \mathbb{C})$. We use decomposition classes to stratify the strongly regular set by subvarieties $X_{\mathcal{D}}$. We construct an étale cover $\hat{\mathfrak{g}}_{\mathcal{D}}$ of $X_{\mathcal{D}}$ and show that $X_{\mathcal{D}}$ and $\hat{\mathfrak{g}}_{\mathcal{D}}$ are smooth and irreducible. We then use Poisson geometry to lift the Gelfand-Zeitlin vector fields on $X_{\mathcal{D}}$ to Hamiltonian vector fields on $\hat{\mathfrak{g}}_{\mathcal{D}}$ and integrate these vector fields to an action of a connected, commutative algebraic group.
\end{abstract}

\section{INTRODUCTION}

In a series of papers KW06a, KW06b, Kostant and Wallach study the action of a complex Lie group $A$ on $\mathfrak{g}=\mathfrak{g l}(n, \mathbb{C})$. The group $A$ is the simply connected, complex Lie group corresponding to the abelian Lie algebra $\mathfrak{a}$ generated by the Hamiltonian vector fields of the Gelfand-Zeitlin collection of functions. The Gelfand-Zeitlin collection of functions contains $\frac{n(n+1)}{2}$ Poisson commuting functions and its restriction to each regular adjoint orbit forms an integrable system. For each function in the collection, the corresponding Hamiltonian vector field is complete. The action of $A$ on $\mathfrak{g}$ is then defined by integrating the Lie algebra $\mathfrak{a}$.

Kostant and Wallach consider a Zariski open subset of $\mathfrak{g}$, called the set of strongly regular elements, which consists of all elements where the differentials of the GelfandZeitlin functions are linearly independent. The $A$-orbits of strongly regular elements are of dimension $\left(\begin{array}{l}n \\ 2\end{array}\right)$ and form Lagrangian submanifolds of regular adjoint orbits. We denote by $x_{i}$ the upper left $i \times i$ corner of the matrix $x \in \mathfrak{g}$. Kostant and Wallach consider the Zariski open subset of strongly regular elements $M_{\Omega}(n)$ consisting of $x \in \mathfrak{g}$ such that each $x_{i}$ is a regular semisimple element of $\mathfrak{g}_{i}$ and $x_{i}$ and $x_{i+1}$ have no common eigenvalues. In [KW06b], they show that there exists a covering $M_{\Omega}(n, \mathfrak{e}) \rightarrow M_{\Omega}(n)$ such that the Lie algebra $\mathfrak{a}$ lifts to $M_{\Omega}(n, \mathfrak{e})$ and integrates to an algebraic action of a torus. Our purpose in this paper is to extend this algebraic integrability result to the full locus of strongly regular elements. More precisely, we stratify the strongly regular set by smooth subvarieties, and for each stratum we construct a covering such that the Lie algebra $\mathfrak{a}$ lifts to the covering and integrates to an algebraic action of a connected, abelian algebraic group.

In more detail, the Gelfand-Zeitlin collection on $\mathfrak{g}$ is the collection of functions $J_{G Z}=$ $\left\{f_{i, j}(x): i=1, \ldots, n, j=1, \ldots, i\right\}$, where $f_{i, j}(x)=\operatorname{Tr}\left(\left(x_{i}\right)^{j}\right)$. We denote by $\mathfrak{g}_{i}=\left\{x_{i}\right.$ : $x \in \mathfrak{g}\} \cong \mathfrak{g l}(i)$ embedded in $\mathfrak{g}$ as the upper left corner, and denote by $G_{i} \cong G L(i)$ the 
corresponding group. The space $\mathfrak{a}$ spanned by $\left\{\xi_{f}: f \in J_{G Z}\right\}$ is an abelian Lie algebra. An element $x \in \mathfrak{g}$ is called strongly regular if $\left\{d f(x): f \in J_{G Z}\right\}$ is linearly independent. Kostant and Wallach showed that the set $\mathfrak{g}_{\text {sreg }}$ of $\mathfrak{g}$ consisting of strongly regular elements is open and Zariski dense. We stratify the strongly regular set using decomposition classes. Let $\mathfrak{l}_{i}$ be a standard Levi subalgebra of $\mathfrak{g}_{i}$ with blocks of sizes $n_{1}, \ldots, n_{k}$, let $\mathfrak{z}_{i}$ be the center of $\mathfrak{l}_{i}$, and let $\mathfrak{z}_{i, g e n}$ be the set of elements in $\mathfrak{z}_{i}$ with centralizer $\mathfrak{l}_{i}$. The regular decomposition class $D_{i}$ in $\mathfrak{g}_{i}$ consists of the subset $G_{i} \cdot\left(\mathfrak{z}_{i, g e n}+e^{i}\right)$, where $e^{i}$ is the unique principal nilpotent element of $\mathfrak{l}_{i}$ in Jordan canonical form. Thus, $D_{i}$ consists of all regular elements of $\mathfrak{g}_{i}$ whose Jordan form has blocks of sizes $n_{1}, \ldots, n_{k}$. Let $W^{L_{i}}=N_{G_{i}}\left(\mathfrak{l}_{i}\right) / L_{i}$, which is a product of symmetric groups and acts on $\mathfrak{l}_{i}$ by permuting blocks of the same size. If $D_{i} \subset \mathfrak{g}_{i} i=1, \ldots, n$ is a sequence of regular decomposition classes, we call the sequence $\mathcal{D}=\left(D_{1}, \ldots, D_{n}\right)$ regular decomposition data.

Let

$$
\begin{gathered}
X_{\mathcal{D}}=\left\{x \in \mathfrak{g}: x_{i} \in D_{i}\right\} \cap \mathfrak{g}_{\text {sreg }}, \\
\hat{\mathfrak{g}}_{\mathcal{D}}=\left\{\left(x, z_{1}, \ldots, z_{n}\right) \in X_{\mathcal{D}} \times \prod_{i=1}^{n} \mathfrak{z}_{i, g e n}: x_{i} \in G_{i} \cdot\left(z_{i}+e^{i}\right)\right\} .
\end{gathered}
$$

Consider the morphism $\mu: \hat{\mathfrak{g}}_{\mathcal{D}} \rightarrow X_{\mathcal{D}}$ given by projection on the first factor, and let $\Sigma_{\mathcal{D}}=\prod_{i=1}^{n} W^{L_{i}}$.

Theorem 1.1. (Theorems 3.10, 3.12 and 5.18) The morphism $\mu: \hat{\mathfrak{g}}_{\mathcal{D}} \rightarrow X_{\mathcal{D}}$ is a $\Sigma_{\mathcal{D}^{-}}$ covering of smooth varieties. Further, $\hat{\mathfrak{g}}_{\mathcal{D}}$ and $X_{\mathcal{D}}$ are connected.

The variety $X_{\mathcal{D}}$ is easily seen to be $A$-invariant, but the Lie algebra $\mathfrak{a}$ integrates to an action of an algebraic group on $X_{\mathcal{D}}$ only for certain special choices of regular decomposition data $\mathcal{D}$ (see Remark 5.14).

Consider the connected, abelian algebraic group $Z_{\mathcal{D}}=\prod_{i=1}^{n-1} Z_{G_{i}}\left(\mathfrak{z}_{i}+e^{i}\right)$.

Theorem 1.2. (Theorem 5.13) The Lie algebra a lifts to $\hat{\mathfrak{g}}_{\mathcal{D}}$, and integrates to a free algebraic action of $Z_{\mathcal{D}}$ on $\hat{\mathfrak{g}}_{\mathcal{D}}$.

Consider the open subsets $X_{\mathcal{D}, \text { gen }}=\left\{x \in X_{\mathcal{D}}: x_{i}\right.$ and $x_{i+1}$ have no common eigenvalues $\}$ and $\hat{\mathfrak{g}}_{\mathcal{D}, \text { gen }}=\mu^{-1}\left(X_{\mathcal{D}, \text { gen }}\right)$. It is easily seen that $Z_{\mathcal{D}}$ acts on $\hat{\mathfrak{g}}_{\mathcal{D}, \text { gen }}$. In the special case where each $D_{i}$ consists of regular semisimple elements of $\mathfrak{g}_{i}$, the covering $\hat{\mathfrak{g}}_{\mathcal{D} \text {,gen }} \rightarrow X_{\mathcal{D}, \text { gen }}$ coincides with the covering $M_{\Omega}(n, \mathfrak{e}) \rightarrow M_{\Omega}(n)$ from [KW06b] and the group $Z_{\mathcal{D}}=\left(\mathbb{C}^{\times}\right)^{\left(\begin{array}{l}n \\ 2\end{array}\right)}$ is a torus, and our result specializes to the algebraic integrability result of Kostant and Wallach from [KW06b] (see Remark 5.19). Thus, Theorem 1.2 generalizes this result to all of $\mathfrak{g}_{\text {sreg }}$, since $\mathfrak{g}_{\text {sreg }}=\bigcup X_{\mathcal{D}}$, where the union is taken over all regular decomposition data $\mathcal{D}$.

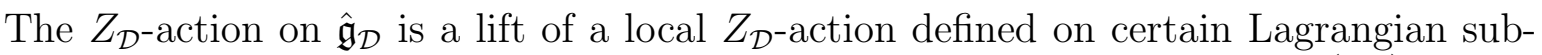
varieties of $X_{\mathcal{D}}$ by the first author in Section 4 of [Col]. Let $\Phi: \mathfrak{g}_{\text {sreg }} \rightarrow \mathbb{C}^{\left(\begin{array}{c}n+1 \\ 2\end{array}\right)}$ be the moment map for the Gelfand-Zeitlin integrable system. In [KW06a], Kostant and Wallach show that for $x \in M_{\Omega}(n), \Phi^{-1}(\Phi(x))$ is a single $A$-orbit and a homogeneous space for a 
free algebraic action of $\left(\mathbb{C}^{\times}\right)^{\left(\begin{array}{l}n \\ 2\end{array}\right)}$. In [Col], the first author describes the action of $A$ on all strongly regular elements. Further, in [Col], for each sequence of regular decomposition data $\mathcal{D}$, an algebraic $Z_{\mathcal{D}^{-}}$action is constructed on the fibres $\Phi^{-1}(\Phi(x))$ for each $x \in X_{\mathcal{D}}$.

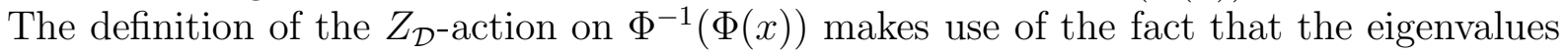
of each $x_{i}, i=1, \ldots, n$ are constant on $\Phi^{-1}(\Phi(x))$. This action of $Z_{\mathcal{D}}$ cannot in general be extended to all of $X_{\mathcal{D}}$, because there is no morphism $X_{\mathcal{D}} \rightarrow \mathbb{C}^{r_{i}}, r_{i}=\operatorname{dim} \mathfrak{z}_{i}$ which assigns to $x_{i}$ a tuple of its eigenvalues in a prescribed order, except for certain regular decomposition data $\mathcal{D}$ (see Remarks 3.13 and 5.14). On the other hand, the covering $\hat{\mathfrak{g}}_{\mathcal{D}}$ has a natural morphism which assigns to each $x_{i}$ an ordered tuple of eigenvalues, namely the morphism $\hat{\mathfrak{g}}_{\mathcal{D}} \rightarrow \mathfrak{z}_{i, \text { gen }}$ which sends $\left(x, z_{1}, \ldots, z_{n}\right) \rightarrow z_{i}$. This allows us to lift the local $Z_{\mathcal{D}}$-action on $\Phi^{-1}(\Phi(x))$ to an algebraic action on $\hat{\mathfrak{g}}_{\mathcal{D}}$.

We construct the lift $\hat{\mathfrak{a}}$ of $\mathfrak{a}$ to $\hat{\mathfrak{g}}_{\mathcal{D}}$ using Poisson geometry. More precisely, $\hat{\mathfrak{g}}_{\mathcal{D}}$ is a subvariety of a Poisson variety $\dot{\mathfrak{g}}_{\mathcal{D}}$. Let $r_{i}=\operatorname{dim}\left(\mathfrak{z}_{i}\right)$ and let $s_{i}=i-r_{i}$. For $\left(x, z_{1}, \ldots, z_{n}\right) \in$ $\hat{\mathfrak{g}}_{\mathcal{D}}$, we use the semisimple part of the Jordan form of $x_{i}$ to construct $r_{i}$ functions $q_{i, j}$ on $\dot{\mathfrak{g}}_{\mathcal{D}}$ (Section 5.1) and the nilpotent part of the Jordan form of $x_{i}$ to construct $s_{i}$ functions $p_{i, k}$ on $\dot{\mathfrak{g}}_{\mathcal{D}}$ (Section [5.2). We let $\hat{\mathfrak{a}}_{i}$ be the span of the Hamiltonian vector fields $\xi_{q_{i, j}}$ and $\xi_{p_{i, k}}$, and we let $\hat{\mathfrak{a}}=\sum_{i=1}^{n-1} \hat{\mathfrak{a}}_{i}$. We show that $\hat{\mathfrak{a}}$ is an abelian Lie algebra of vector fields tangent to $\hat{\mathfrak{g}}_{\mathcal{D}}$ (Proposition 5.10), and further show that $\mu_{*}(\hat{\mathfrak{a}})=\mathfrak{a}$ (Lemma 5.11). In addition, the vector fields $\xi_{q_{i, j}}$ integrate to give an algebraic $\mathbb{C}^{\times}$-action on $\hat{\mathfrak{g}}_{\mathcal{D}}$ (Proposition 5.4), and the vector fields $\xi_{p_{i, k}}$ integrate to give an algebraic $\mathbb{C}$-action on $\hat{\mathfrak{g}}_{\mathcal{D}}$ (Proposition 5.9). These results imply that $\hat{\mathfrak{a}}$ integrates to give an algebraic action of $Z_{\mathcal{D}}$ on $\hat{\mathfrak{g}}_{\mathcal{D}}$. The $Z_{\mathcal{D}^{-}}$action on $\hat{\mathfrak{g}}_{\mathcal{D}}$ is given by a simple formula, and projects and specializes to the more complicated $Z_{\mathcal{D}^{\text {-action }}}$ defined on the fibers $\Phi^{-1}(\Phi(x)), x \in X_{\mathcal{D}}$. We regard the simplicity of the $Z_{\mathcal{D}}$-action on $\hat{\mathfrak{g}}_{\mathcal{D}}$ as a useful feature of the cover $\hat{\mathfrak{g}}_{\mathcal{D}}$. In addition, the cover $\hat{\mathfrak{g}}_{\mathcal{D}}$ facilitates the use of Jordan decomposition to separate the flows into semisimple and nilpotent parts, and makes Poisson computations easy to do, as in [KKS78] and in [EL07]. It would be interesting to relate our work to work of Bielawski and Pidstrygach [BP08], where a different approach to the geometry of the Gelfand-Zeitlin action is taken.

The paper is organized as follows. In Section 2, we recall results from [KW06a and [KW06b], as well as the thesis of the first author [Col07], Col]. In Section 3, we recall facts about decomposition classes, show $X_{\mathcal{D}}$ is smooth, and construct the covering $\hat{\mathfrak{g}}_{\mathcal{D}} \rightarrow X_{\mathcal{D}}$. In Section 4, we construct a Poisson structure on $\dot{\mathfrak{g}}_{\mathcal{D}}$ and compute its anchor map. In Section 5, we prove the main results of the paper.

In this paper, a variety is a complex quasi-projective algebraic set, and a subset of a variety is called a subvariety if it is locally closed. The ring of regular functions on a variety $Y$ is denoted $\mathbb{C}[Y]$. We use the Zariski topology on a variety unless otherwise stated.

We would like to thank Michael Gekhtman, Bert Kostant, Hanspeter Kraft, Nolan Wallach, and Milen Yakimov for useful conversations relevant to the subject of this paper. The second author was partially supported by NSA grant H98230-08-1-0023 during the preparation of this paper. 


\section{Notation and Results of Kostant, Wallach, and Colarusso}

Let $\mathfrak{g}=\mathfrak{g l}(n, \mathbb{C})$ be the Lie algebra of $n \times n$ complex matrices. For $i \leq n$, let $\mathfrak{g}_{i}=$ $\mathfrak{g l}(i, \mathbb{C}) \subset \mathfrak{g}$, regarded as the upper left $i \times i$ corner. If $x \in \mathfrak{g}$, let $x_{i}$ be its upper left $i \times i$ corner of $x$, so the $k j$ matrix coefficient $\left(x_{i}\right)_{k j}$ of $x_{i}$ is $(x)_{k j}$ if $1 \leq k, j \leq i$, and is zero otherwise. Let $G_{i} \cong G L(i, \mathbb{C})$ be the closed Lie subgroup of $G L(n, \mathbb{C})$ with Lie algebra $\mathfrak{g}_{i}$.

The projection $\mathfrak{g} \rightarrow \mathfrak{g}_{i}$ given by $x \mapsto x_{i}$ induces an injective ring homomorphism $\mathbb{C}\left[\mathfrak{g}_{i}\right] \rightarrow \mathbb{C}[\mathfrak{g}]$, which we use to regard $\mathbb{C}\left[\mathfrak{g}_{i}\right]$ as a subalgebra of $\mathbb{C}[\mathfrak{g}]$. In particular, we regard $J(n)=\mathbb{C}\left[\mathfrak{g}_{1}\right]^{G_{1}} \otimes_{\mathbb{C}} \cdots \otimes_{\mathbb{C}} \mathbb{C}\left[\mathfrak{g}_{n}\right]^{G_{n}}$ as a subalgebra of $\mathbb{C}[\mathfrak{g}]$.

For $i \leq n$ and $j=1, \ldots, i$, let $f_{i, j} \in \mathbb{C}[\mathfrak{g}]$ be the regular function defined by $f_{i, j}=$ $\operatorname{Tr}\left(\left(x_{i}\right)^{j}\right)$. Note that $f_{i, j} \in \mathbb{C}\left[\mathfrak{g}_{i}\right]^{G L(i)} \subset J(n)$. Let $J_{G Z}=\left\{f_{i, j}: 1 \leq i \leq n, 1 \leq j \leq i\right\}$. Then $J_{G Z}$ freely generates the polynomial algebra $J(n)$. Let $\mathfrak{a}=\operatorname{span}\left\{\xi_{f}: f \in J_{G Z}\right\}$, where $\xi_{f}$ is the Hamiltonian vector field on $\mathfrak{g}$ defined by a function $f \in \mathbb{C}[\mathfrak{g}]$ using the Lie-Poisson Poisson structure on $\mathfrak{g}$. It is shown in Theorem 3.25 of [KW06a] that $J(n)$ is a maximal Poisson commutative subalgebra of $\mathbb{C}[\mathfrak{g}]$. Thus, $\mathfrak{a}$ is an abelian Lie algebra, and further $\operatorname{dim}(\mathfrak{a})=\left(\begin{array}{l}n \\ 2\end{array}\right)$ (see [KW06a], Section 3.2). Further, the vector fields $\xi_{f_{n, j}}=0$, so $\mathfrak{a}$ is spanned by the vector fields $\xi_{f_{i, j}}$ with $i \leq n-1$. Let $A$ be the simply connected holomorphic Lie group with Lie algebra $\mathfrak{a}$. By Section 3 of [KW06a], the group $A \cong \mathbb{C}\left(\begin{array}{l}n \\ 2\end{array}\right)$ integrates the action of $\mathfrak{a}$ on $\mathfrak{g}$. It follows from standard results in symplectic geometry that $A \cdot x$ is isotropic in the symplectic leaf $G \cdot x$ in $\mathfrak{g}$.

By definition, $x \in \mathfrak{g}$ is called strongly regular if the set $\left\{d f(x): f \in J_{G Z}\right\}$ is linearly independent in $T_{x}^{*}(\mathfrak{g})$. Let $\mathfrak{g}_{\text {sreg }}$ be the set of strongly regular elements of $\mathfrak{g}$ and let $\mathfrak{g}_{\text {reg }}$ be the set of regular elements of $\mathfrak{g}$, i.e., the set of elements whose centralizer $\mathfrak{z} \mathfrak{g}(x)$ has dimension $n$. By a well-known result of Kostant [Kos63], if $x \in \mathfrak{g}_{\text {sreg }}, x_{k}$ is regular for all $k$ ([KW06a], Proposition 2.6).

We give alternate characterizations of the strongly regular set in $\mathfrak{g}$.

Theorem 2.1. [KW06a Let $x \in \mathfrak{g}$. Then the following are equivalent.

(1) $x$ is strongly regular.

(2) $\operatorname{dim}(A \cdot x)=\operatorname{dim}(A)=\left(\begin{array}{l}n \\ 2\end{array}\right)$ and $A \cdot x$ is Lagrangian in $G \cdot x$.

(3) $x_{i} \in \mathfrak{g}_{i}$ is regular for all $i, 1 \leq i \leq n$ and $\mathfrak{z}_{\mathfrak{g}_{i}}\left(x_{i}\right) \cap \mathfrak{z} \mathfrak{g}_{i+1}\left(x_{i+1}\right)=0$ for all $1 \leq i \leq n-1$.

Further, let $\mathfrak{b}$ be the upper triangular Borel subalgebra of $\mathfrak{g}$, and let $e$ be the standard level one regular nilpotent in the opposite Borel to $\mathfrak{b}$, i.e., $(e)_{k+1, k}=1$, and $(e)_{l, k}=0$ if $l \neq k+1$. Then $\mathfrak{b}+e$ is contained in the strongly regular set $\mathfrak{g}_{\text {sreg }}$. Elements in the variety $\mathfrak{b}+e$ are called (upper) Hessenberg matrices. It follows that $\mathfrak{g}_{\text {sreg }}$ is Zariski open and dense in $\mathfrak{g}$.

For $i=1, \ldots, n$, consider the morphisms

$$
\Phi_{i}: \mathfrak{g}_{i} \rightarrow \mathbb{C}^{i}, \Phi_{i}(y)=\left(p_{i, 1}(y), \ldots, p_{i, i}(y)\right)
$$


where $p_{i, j}(y)$ is the coefficient of $t^{j-1}$ in the characteristic polynomial of $y$. Note that $p_{i, j} \in \mathbb{C}\left[\mathfrak{g}_{i}\right]^{G L(i)}$. Define

$$
\Phi: \mathfrak{g} \rightarrow \mathbb{C}^{1} \times \cdots \times \mathbb{C}^{n}=\mathbb{C}^{\left(\begin{array}{c}
n+1 \\
2
\end{array}\right)}, \Phi(x)=\left(\Phi_{1}\left(x_{1}\right), \ldots, \Phi_{n}\left(x_{n}\right)\right) .
$$

Then the Kostant-Wallach map $\Phi: \mathfrak{b}+e \rightarrow \mathbb{C}^{\left(\begin{array}{c}n+1 \\ 2\end{array}\right)}$ is an isomorphism of varieties ([KW06a], Theorem 2.3). Hence, for $c \in \mathbb{C}^{\left(\begin{array}{c}n+1 \\ 2\end{array}\right), \Phi^{-1}(c)}$ sreg $:=\Phi^{-1}(c) \cap \mathfrak{g}_{\text {sreg }}$ is nonempty and open. By Proposition 3.6 in [KW06a, $A \cdot x \subset \Phi^{-1}(c)$.

For $x \in \mathfrak{g}_{i}$, let $\sigma_{i}(x)$ equal the collection of $i$ eigenvalues of $x$ counted with repetitions, where here we regard $x$ as an $i \times i$ matrix.

Remark 2.2. If $x, y \in \mathfrak{g}$, then $\Phi(x)=\Phi(y)$ if and only if $\sigma_{i}\left(x_{i}\right)=\sigma_{i}\left(y_{i}\right)$ for $i=1, \ldots, n$.

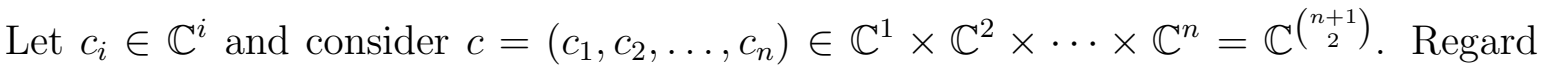
$c_{i}=\left(z_{1}, \ldots, z_{i}\right)$ as the coefficients of the degree $i$ monic polynomial

$$
p_{c_{i}}(t)=z_{1}+z_{2} t+\cdots+z_{i} t^{i-1}+t^{i} .
$$

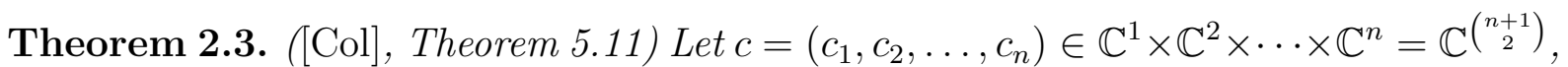
and suppose that $p_{c_{i}}(t)$ and $p_{c_{i+1}}(t)$ have exactly $j_{i}$ roots in common. Then there are exactly $2^{\sum_{i=1}^{n-1} j_{i}}$ distinct $A$-orbits in $\Phi^{-1}(c)_{\text {sreg. }}$. For $x \in \Phi^{-1}(c)_{\text {sreg }}$, let $Z_{D_{i}}$ denote the centralizer of the Jordan form of $x_{i}$ in $\mathfrak{g}_{i}$, and consider the abelian connected algebraic group $Z_{\mathcal{D}}=Z_{D_{1}} \times \cdots \times Z_{D_{n-1}}$. Then $Z_{\mathcal{D}}$ acts freely and algebraically on $\Phi^{-1}(c)_{\text {sreg }}$, and

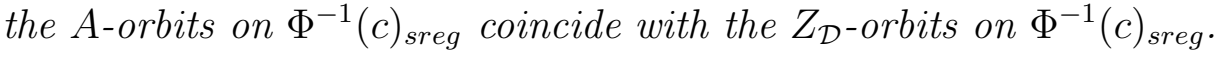

\section{DeComposition Classes AND DECOMPOSITION TOWERS}

3.1. Decomposition classes. We recall some results about decomposition classes. See the papers [Bro98b] and Bro98a of Broer for details. Let $\mathfrak{l}$ be a Levi subalgebra of a reductive Lie algebra $\mathfrak{k}$, let $\mathfrak{z}$ be the center of $\mathfrak{l}$, and let $W^{L}:=N_{K}(\mathfrak{l}) / L$, where $K$ is the adjoint group of $\mathfrak{k}$. Let $\mathfrak{z}_{\text {gen }}=\{z \in \mathfrak{z}: \mathfrak{z} \mathfrak{k}(z)=\mathfrak{l}\}$, and let $\mathcal{O}_{x}$ be the $L$-orbit through nilpotent $x \in \mathfrak{l}$. We say that the decomposition class in $\mathfrak{k}$ associated to $\mathfrak{l}$ and $x$ is the set

$$
D(\mathfrak{l}, x):=K \cdot\left(\mathfrak{z}_{\text {gen }}+\mathcal{O}_{x}\right)=K \cdot\left(\mathfrak{z}_{\text {gen }}+x\right) .
$$

By [Bro98a], Proposition 2.3, the morphism

$$
K \times_{N_{K}(\mathfrak{l})}\left(\mathfrak{z}_{\text {gen }}+N_{K}(\mathfrak{l}) \cdot x\right) \cong D(\mathfrak{l}, x),(k, y) \mapsto \operatorname{Ad}(k) y
$$

is an isomorphism, so in particular $D(\mathfrak{l}, x)$ is smooth. Let

$$
\dot{D}(\mathfrak{l}, x):=K \times_{L}\left(\mathfrak{z}_{\text {gen }}+N_{K}(\mathfrak{l}) \cdot x\right),
$$

and consider the étale morphism $\mu: \dot{D}(\mathfrak{l}, x) \rightarrow D(\mathfrak{l}, x)$ given by $\mu(g, y)=\operatorname{Ad}(g) y$. By [Bro98a], Proposition 2.3(iii), the surjective morphism $\theta: \dot{D}(\mathfrak{l}, x) \rightarrow \mathfrak{z}_{\text {gen }}$ given by $\theta(g, z+$ $y)=z$ for $g \in G, z \in \mathfrak{z}_{\text {gen }}$ and $y \in N_{K}(\mathfrak{l}) \cdot x$ descends to give a surjective morphism $\bar{\theta}: D(\mathfrak{l}, x) \rightarrow \mathfrak{z}_{\text {gen }} / W^{L}$. Denote by $q: \mathfrak{z}_{\text {gen }} \rightarrow \mathfrak{z}_{\text {gen }} / W^{L}$ the quotient morphism. 
Proposition 3.1. (See [Bro98a], Proposition 2.3) Let $D(\mathfrak{l}, x)$ be a decomposition class.

(1) The following diagram is Cartesian with étale horizontal maps:

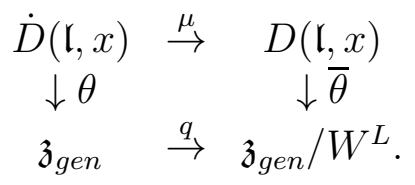

In particular, $\dot{D}(\mathfrak{l}, x)$ is smooth.

(2) $\bar{\theta}(D(\mathfrak{l}, x))=\mathfrak{z}_{\text {gen }} / W^{L}$ is smooth.

Remark 3.2. Let

$$
\mathcal{D}_{c}(\mathfrak{l}, x)=D(\mathfrak{l}, x) \times_{\mathfrak{z}_{\text {gen }} / W^{L}} \mathfrak{z}_{\text {gen }}=\left\{(y, z): y \in K \cdot\left(z+N_{K}(\mathfrak{l}) \cdot x\right), z \in \mathfrak{z}_{\text {gen }}\right\} .
$$

By Proposition [3.1, the morphism $\beta: \dot{D}(\mathfrak{l}, x) \rightarrow \mathcal{D}_{c}(\mathfrak{l}, x)$ given by $\beta(g, z+y)=(\operatorname{Ad}(g)(z+$ $y)$, z) for $g \in K, z \in \mathfrak{z}_{\text {gen }}$, and $y \in N_{K}(\mathfrak{l}) \cdot x$ is an isomorphism.

Remark 3.3. Let $r$ be the rank of $\mathfrak{k}$ and let $f_{1}, \ldots, f_{r}$ be algebraically independent generators of $\mathbb{C}[\mathfrak{k}]^{K}$, and consider the morphism $F: \mathfrak{k} \rightarrow \mathbb{C}^{r}$ given by $F(x)=\left(f_{1}(x), \ldots, f_{r}(x)\right)$. Then if $\chi: \mathfrak{k} \rightarrow \mathfrak{k} / / K$ is the geometric invariant theory quotient, there is an induced isomorphism $\bar{F}: \mathfrak{k} / / K \rightarrow \mathbb{C}^{r}$. Let $\mathfrak{h} \subset \mathfrak{g}$ be a Cartan subalgebra containing $\mathfrak{z}_{\text {gen }}$ and let $W=N_{G}(\mathfrak{h}) / H$ be the Weyl group. Then, the diagram

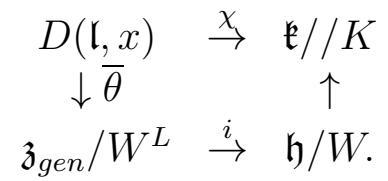

commutes, where $i$ is the embedding induced by the inclusion $\mathfrak{z}_{\text {gen }} \rightarrow \mathfrak{h}$, and the right vertical arrow is the Chevalley isomorphism. As a consequence, $F(D(\mathfrak{l}, x)) \cong \chi(D(\mathfrak{l}, x)) \cong$ $\bar{\theta}(D(\mathfrak{l}, x))$ is smooth by Proposition [3.1. We will apply these ideas later with $\mathfrak{k}=\mathfrak{g}_{i}$, and $F=\Phi_{i}: \mathfrak{g}_{i} \rightarrow \mathbb{C}^{i}$ as in Section Q

Remark 3.4. We say a decomposition class $D$ is regular if it is contained in $\mathfrak{k}_{\text {reg }}$, the set of regular elements of $\mathfrak{k} . D(\mathfrak{l}, x)$ is regular if and only if $x$ is regular nilpotent in $\mathfrak{l}$. In the remainder of this paper, we consider only regular $D(\mathfrak{l}, x)$, in which case $N_{K}(\mathfrak{l}) \cdot x=L \cdot x$.

Remark 3.5. In the case when $\mathfrak{k}=\mathfrak{g l}(n)$, a regular decomposition class $D$ corresponds to a partition of $n$. Indeed, if $\lambda=\left(n_{1}, \ldots, n_{r}\right)$ is a partition of $n$, we associate to $\lambda$ the Levi subalgebra $\mathfrak{l}$ consisting of block diagonal matrices with blocks of size $n_{j}$ for $j=1, \ldots, r$. The corresponding decomposition class consists of matrices conjugate to a block diagonal matrix $M(\lambda)$ with blocks of size $n_{j}$ for $j=1, \ldots, r$, where the $j$ th block $M_{j}=z_{j} \mathrm{id}_{n_{j}}+x^{j}$, where $\operatorname{id}_{n_{j}}$ is the $n_{j} \times n_{j}$ identity matrix, and $x^{j}$ is a regular nilpotent element of $\mathfrak{g l}\left(n_{j}\right)$, and $z_{i} \neq z_{j}$ if $i \neq j$. It is elementary to show that every regular element of $\mathfrak{g l}(n)$ is in $M(\lambda)$ for some partition $\lambda$. The group $W^{L}$ is a product of symmetric groups, given by permuting blocks of the same size ([Bro98a], Section 9.1). 
Lemma 3.6. Let $x, y \in \mathfrak{g}_{\text {sreg }}$. If $\Phi(x)=\Phi(y)$, then $y_{i} \in G_{i} \cdot x_{i}$ for $i=1, \ldots, n$.

Proof. Since $\Phi_{i}(x)=\Phi_{i}(y)$, then $\chi\left(x_{i}\right)=\chi\left(y_{i}\right)$, where $\chi: \mathfrak{g l}(i) \rightarrow \mathfrak{g l}(i) / / G L(i)$ is the adjoint quotient. The lemma now follows since each fiber of the adjoint quotient has a unique regular conjugacy class ([Kos63]).

Q.E.D.

Let $P$ be a parabolic subgroup of $K$ with Levi factor $L$ and unipotent radical $U$, let $\mathfrak{u}$ and $\mathfrak{p}$ be the corresponding Lie algebras, and let $\mathfrak{l}_{1}=[\mathfrak{l}, \mathfrak{l}]$. Note that the quotient morphism $\beta: \dot{D}(\mathfrak{l}, x) \rightarrow K \times_{P}\left(\mathfrak{z}_{\text {gen }}+L \cdot x+\mathfrak{u}\right)$ is an isomorphism. Surjectivity follows from the observation that if $z \in \mathfrak{z}_{\text {gen }}$ and $y$ is nilpotent in $\mathfrak{l}_{1}$, then $U \cdot(z+y)=z+y+\mathfrak{u}$. The reader may verify this assertion using the fact that $z+y+\mathfrak{u}$ is an irreducible $U$ variety, $U \cdot(x+y)$ is closed in $z+y+\mathfrak{u}$, and the stabilizer $U_{z+y}$ is trivial. The remaining steps are routine to verify. In particular, we regard $\dot{D}(\mathfrak{l}, x)$ as a locally closed subvariety of $\dot{\mathfrak{k}}:=K \times_{P} \mathfrak{p}$.

Let $\tilde{\mathfrak{k}}:=K / P \times \mathfrak{k}$, and note that the morphism $\alpha: \dot{\mathfrak{k}} \rightarrow \tilde{\mathfrak{k}}$ given by $\alpha(g, y)=(g P, \operatorname{Ad}(g) y)$ is a closed embedding.

3.2. Decomposition towers. For $i=1, \ldots, n$, choose a regular decomposition class $D_{i}=G_{i} \cdot\left(\mathfrak{z}_{i, \text { gen }}+\mathcal{O}_{u_{i}}\right)$, with $\mathfrak{z}_{i, g e n}$ the generic part of the center $\mathfrak{z}_{i}$ of the Levi factor $\mathfrak{l}_{i}$ of $\mathfrak{g}_{i}$ determined by $D_{i}$, and $\mathcal{O}_{u_{i}}$ the regular nilpotent $L_{i}$-orbit in $\mathfrak{l}_{i}$. Let $P_{i}$ be a parabolic containing $L_{i}$ for $i=1, \ldots, n$. We call the collection $\mathcal{D}=\left(D_{1}, \ldots, D_{n}\right)$ regular decomposition data. Let

$$
\Sigma_{\mathcal{D}}=W^{L_{1}} \times \cdots \times W^{L_{n}} .
$$

The group $\Sigma_{\mathcal{D}}$ is a product of symmetric groups (see [Bro98a], Section 9.1). Let

$$
\mathfrak{z}_{\mathcal{D}}:=\mathfrak{z}_{1, \text { gen }} \oplus \cdots \oplus \mathfrak{z}_{n, \text { gen }}
$$

and note that the product action of $\Sigma_{\mathcal{D}}$ on $\mathfrak{z}_{\mathcal{D}}$ is free, so $\mathfrak{z}_{\mathcal{D}} / \Sigma_{\mathcal{D}}$ is smooth of dimension $\operatorname{dim}\left(\mathfrak{z}_{\mathcal{D}}\right)$.

Definition 3.7. The subvariety

$$
X_{\mathcal{D}}:=\left\{x \in \mathfrak{g}_{\text {sreg }}: x_{i} \in D_{i}\right\}
$$

is called a tower of decomposition classes.

Recall the morphisms $\overline{\theta_{i}}: D_{i} \rightarrow \mathfrak{z}_{i, g e n} / W^{L_{i}}$ from Diagram (3.1). Denote by $\overline{\theta_{\mathcal{D}}}: X_{\mathcal{D}} \rightarrow$ $\mathfrak{z}_{\mathcal{D}} / \Sigma_{\mathcal{D}}$ the morphism $\overline{\theta_{\mathcal{D}}}(x)=\left(\overline{\theta_{1}}\left(x_{1}\right), \ldots, \overline{\theta_{n}}\left(x_{n}\right)\right)$. Let $\mathfrak{h}_{i}$ be a Cartan subalgebra of $\mathfrak{g}_{i}$ containing $\mathfrak{z}_{i}$, and let $W_{i}$ be $N_{G_{i}}\left(\mathfrak{h}_{i}\right) / H_{i}$, the corresponding Weyl group. Recall the embedding $\mathfrak{z}_{i, g e n} / W^{L_{i}} \rightarrow \mathfrak{h}_{i} / W_{i}$ and the isomorphism $\Phi_{i}: \mathfrak{h}_{i} / W_{i} \rightarrow \mathbb{C}^{i}$ from Remark 3.3. 
They compose to give an embedding $\overline{\Phi_{i}}: \mathfrak{z}_{i, g e n} / W^{L_{i}} \rightarrow \mathbb{C}^{i}$. We consider the product morphism

$$
\bar{\Phi}=\left(\overline{\Phi_{1}}, \ldots, \overline{\Phi_{n}}\right): \mathfrak{z}_{\mathcal{D}} / \Sigma_{\mathcal{D}} \rightarrow \mathbb{C}^{\left(\begin{array}{c}
n+1 \\
2
\end{array}\right)}
$$

Lemma 3.8. The diagram

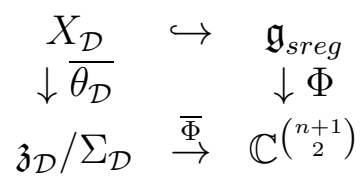

is Cartesian. In particular, $\Phi^{-1}\left(\Phi\left(X_{\mathcal{D}}\right)\right) \cap \mathfrak{g}_{\text {sreg }}=X_{\mathcal{D}}$, so that $X_{\mathcal{D}}$ is a union of $A$-orbits in $\mathfrak{g}_{\text {sreg }}$.

Proof. Let $z=\left(z_{1}, \ldots, z_{n}\right) \in \mathfrak{z}_{\mathcal{D}}$, and denote by $\bar{z}$ its representative in $\mathfrak{z}_{\mathcal{D}} / \Sigma_{\mathcal{D}}$. If $\bar{\Phi}(\bar{z})=$ $\Phi(x)$ for $x \in \mathfrak{g}_{\text {sreg }}$, then $\Phi_{i}\left(z_{i}\right)=\Phi_{i}\left(x_{i}\right)$ for $i=1, \ldots, n$. Thus, $\Phi_{i}\left(z_{i}+u_{i}\right)=\Phi_{i}\left(x_{i}\right)$, so for each $i, x_{i} \in G_{i} \cdot\left(z_{i}+u_{i}\right)$ by the proof of Lemma 3.6. It follows that $x \in X_{\mathcal{D}}$ and that $\overline{\theta_{\mathcal{D}}}(x)=\bar{z}$.

Q.E.D.

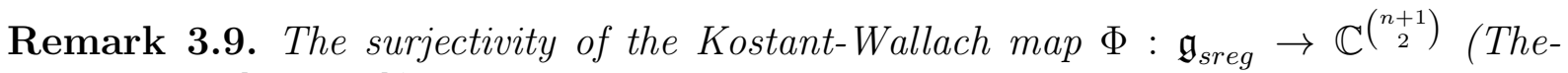
orem 2.3 in [KW06a]) along with the argument in the proof of Lemma 3.8 imply that the morphism $\overline{\theta_{\mathcal{D}}}: X_{\mathcal{D}} \rightarrow \mathfrak{z}_{\mathcal{D}} / \Sigma_{\mathcal{D}}$ is surjective. It then follows easily that $\operatorname{dim} \Phi\left(X_{\mathcal{D}}\right)=$ $\operatorname{dim} \mathfrak{z}_{\mathcal{D}} / \Sigma_{\mathcal{D}}=\operatorname{dim} \mathfrak{z}_{\mathcal{D}}$.

Theorem 3.10. The subvariety $X_{\mathcal{D}}$ is a smooth subvariety of $\mathfrak{g}$, and all its connected components have dimension $\operatorname{dim}\left(\mathfrak{z}_{\mathcal{D}}\right)+n^{2}-\left(\begin{array}{c}n+1 \\ 2\end{array}\right)$.

Proof. By Theorem 2.3 of [KW06a], $\Phi: \mathfrak{g}_{\text {sreg }} \rightarrow \mathbb{C}^{\left(\begin{array}{c}n+1 \\ 2\end{array}\right)}$ is a surjective submersion, so $\Phi$ is smooth of relative dimension $n^{2}-\left(\begin{array}{c}n+1 \\ 2\end{array}\right)$ by Proposition III.10.4 of [Har77]. By Proposition III.10.1(b) of [Har77] and Lemma 3.8, the morphism $\overline{\theta_{\mathcal{D}}}: X_{\mathcal{D}} \rightarrow \mathfrak{z}_{\mathcal{D}} / \Sigma_{\mathcal{D}}$ is smooth of relative dimension $n^{2}-\left(\begin{array}{c}n+1 \\ 2\end{array}\right)$. By Remark 3.9, $\mathfrak{z}_{\mathcal{D}} / \Sigma_{\mathcal{D}}$ is smooth of dimension $\operatorname{dim}\left(\mathfrak{z}_{\mathcal{D}}\right)=\operatorname{dim}\left(\Phi\left(X_{\mathcal{D}}\right)\right)$, and it follows from Proposition III.10.1(c) of Har77 that $X_{\mathcal{D}}$ is smooth of dimension $n^{2}-\left(\begin{array}{c}n+1 \\ 2\end{array}\right)+\operatorname{dim}\left(\mathfrak{z}_{\mathcal{D}}\right)$. The result now follows from definitions.

Q.E.D.

3.3. Covers of decomposition towers. Fix regular decomposition data $\mathcal{D}=\left(D_{1}, \ldots, D_{n}\right)$ and associated notation, as in the last section. Let

$$
\dot{\mathfrak{g}}_{\mathcal{D}}:=\prod_{i=1}^{n} \dot{D}\left(\mathfrak{l}_{i}, u_{i}\right), \quad \tilde{\mathfrak{g}}_{\mathcal{D}}:=\prod_{i=1}^{n} G_{i} / P_{i} \times \mathfrak{g}_{i} .
$$

Consider the locally closed embedding

$$
\alpha=\left(\alpha_{1}, \ldots, \alpha_{n}\right): \dot{\mathfrak{g}}_{\mathcal{D}} \rightarrow \tilde{\mathfrak{g}}_{\mathcal{D}}
$$


where $\alpha_{i}\left(g_{i}, y^{i}\right)=\left(g_{i} P_{i}, \operatorname{Ad}\left(g_{i}\right) y^{i}\right)$ with $g_{i} \in G_{i}$ and $y_{i} \in \mathfrak{z}_{i, g e n}+\mathcal{O}_{u_{i}}$.

Denote by $\mu=\left(\mu_{1}, \ldots, \mu_{n}\right): \dot{\mathfrak{g}}_{\mathcal{D}} \rightarrow \Pi_{i=1}^{n} D_{i}$, where $\mu_{i}\left(g_{i}, y^{i}\right)=\operatorname{Ad}\left(g_{i}\right) y^{i}$. Consider also the embedding $\gamma: \mathfrak{g} \rightarrow \prod_{i=1}^{n} \mathfrak{g}_{i}$ given by $\gamma(x)=\left(x_{1}, \ldots, x_{n}\right)$.

Let $\hat{\mathfrak{g}}_{\mathcal{D}}:=X_{\mathcal{D}} \times_{\Pi_{i=1}^{n} D_{i}} \dot{\mathfrak{g}}_{\mathcal{D}}$, so the diagram

$$
\begin{array}{llc}
\hat{\mathfrak{g}}_{\mathcal{D}} & \rightarrow & \dot{\mathfrak{g}}_{\mathcal{D}} \\
\downarrow \mu & & \downarrow \mu \\
X_{\mathcal{D}} & \stackrel{\gamma}{\rightarrow} & \Pi_{i=1}^{n} D_{i}
\end{array}
$$

is Cartesian. Note that the canonical morphism $\hat{\mathfrak{g}}_{\mathcal{D}} \rightarrow \dot{\mathfrak{g}}_{\mathcal{D}}$ is a locally closed embedding, so we can view $\hat{\mathfrak{g}}_{\mathcal{D}}$ as a subvariety of $\dot{\mathfrak{g}}_{\mathcal{D}}$.

Remark 3.11. A point $y=\left(g_{1}, y^{1}, \ldots, g_{n}, y^{n}\right) \in \dot{\mathfrak{g}}_{\mathcal{D}}$ is contained in $\hat{\mathfrak{g}}_{\mathcal{D}}$ if and only if $\left(\operatorname{Ad}\left(g_{n}\right) y^{n}\right)_{i}=\operatorname{Ad}\left(g_{i}\right) y^{i}$ for $i=1, \ldots, n-1$ and $\operatorname{Ad}\left(g_{n}\right) y^{n} \in \mathfrak{g}_{\text {sreg }}$.

Recall the morphisms $\theta_{i}: \dot{D}\left(\mathfrak{l}_{i}, u_{i}\right) \rightarrow \mathfrak{z}_{i, \text { gen }}, \overline{\theta_{i}}: D_{i} \rightarrow \mathfrak{z}_{i, g e n} / W^{L_{i}}$ from Diagram (3.1). Denote by $\theta_{\mathcal{D}}: \dot{\mathfrak{g}}_{\mathcal{D}} \rightarrow \mathfrak{z}_{\mathcal{D}}$ the morphism $\theta_{\mathcal{D}}=\left(\theta_{1}, \ldots, \theta_{n}\right)$. Abusing notation, we denote by $\overline{\theta_{\mathcal{D}}}: \prod_{i=1}^{n} D_{i} \rightarrow \mathfrak{z}_{\mathcal{D}} / \Sigma_{\mathcal{D}}$ the morphism $\overline{\theta_{\mathcal{D}}}=\left(\overline{\theta_{1}}, \ldots, \overline{\theta_{n}}\right)$. We note that $\overline{\theta_{\mathcal{D}}} \circ \gamma: X_{\mathcal{D}} \rightarrow \mathfrak{z}_{\mathcal{D}} / \Sigma_{\mathcal{D}}$ coincides with the morphism $\overline{\theta_{\mathcal{D}}}: X_{\mathcal{D}} \rightarrow \mathfrak{z}_{\mathcal{D}} / \Sigma_{\mathcal{D}}$ from Section 3.2 ,

Theorem 3.12. The morphism $\mu: \hat{\mathfrak{g}}_{\mathcal{D}} \rightarrow X_{\mathcal{D}}$ is a $\Sigma_{\mathcal{D}}$-covering, $\hat{\mathfrak{g}}_{\mathcal{D}}$ is smooth, and all its connected components have dimension $\operatorname{dim}\left(\mathfrak{z}_{\mathcal{D}}\right)+n^{2}-\left(\begin{array}{c}n+1 \\ 2\end{array}\right)$.

Proof. Consider the Cartesian diagram

$$
\begin{array}{cccc}
\dot{\mathfrak{g}}_{\mathcal{D}} & \stackrel{\theta_{\mathcal{D}}}{\rightarrow} & \mathfrak{z}_{\mathcal{D}} \\
\downarrow \mu & & \downarrow q_{\mathcal{D}} \\
\prod_{i=1}^{n} D_{i} & \stackrel{\overline{\theta_{\mathcal{D}}}}{\rightarrow} & \mathfrak{z}_{\mathcal{D}} / \Sigma_{\mathcal{D}},
\end{array}
$$

where $q_{\mathcal{D}}$ is the quotient of the action of $\Sigma_{\mathcal{D}}$. This diagram is the product of Cartesian diagrams from Equation (3.1). By Proposition 3.1, the product morphism $\mu$ is a $\Sigma_{\mathcal{D}^{-}}$ covering, and the first claim follows easily from the Cartesian diagram (3.3). Smoothness of $\hat{\mathfrak{g}}_{\mathcal{D}}$ and the dimension assertion follow from Theorem 3.10 .

\section{Q.E.D.}

Remark 3.13. Let $\mathcal{D}=\left(D_{1}, \ldots, D_{n}\right)$ with $D_{i}=\left(\mathfrak{l}_{i}, u_{i}\right)$ and suppose for each $i$ that all blocks of $\mathfrak{l}_{i}$ have different sizes (see Remark 3.5). Then $\Sigma_{\mathcal{D}}$ is trivial and $\hat{\mathfrak{g}}_{\mathcal{D}} \cong X_{\mathcal{D}}$.

Remark 3.14. We give another characterization of the variety $\hat{\mathfrak{g}}_{\mathcal{D}}$, which will be useful in Sections 5.4 and 5.5. Indeed, by Diagrams (3.3) and (3.4), it follows that

$$
\begin{array}{ccc}
\hat{\mathfrak{g}}_{\mathcal{D}} & \stackrel{\theta_{\mathcal{D}}}{\rightarrow} & \mathfrak{z}_{\mathcal{D}} \\
\downarrow \mu & & \downarrow q_{\mathcal{D}} \\
X_{\mathcal{D}} & \stackrel{\theta_{\mathcal{D}}}{\rightarrow} & \mathfrak{z}_{\mathcal{D}} / \Sigma_{\mathcal{D}}
\end{array}
$$


is Cartesian. Since $X_{\mathcal{D}} \rightarrow \mathfrak{z}_{\mathcal{D}} / \Sigma_{\mathcal{D}}$ is a surjective submersion, it follows that $\hat{\mathfrak{g}}_{\mathcal{D}} \rightarrow \mathfrak{z}_{\mathcal{D}}$ is a surjective submersion. Hence,

$$
\hat{\mathfrak{g}}_{\mathcal{D}} \cong X_{\mathcal{D}} \times_{\mathfrak{z}_{\mathcal{D}} / \Sigma_{\mathcal{D}}} \mathfrak{z}_{\mathcal{D}}=\mathcal{D}_{c}
$$

where $\mathcal{D}_{c}:=\left\{\left(x, z_{1}, \ldots, z_{n}\right): x \in \mathfrak{g}_{\text {sreg }}, x_{i} \in G_{i} \cdot\left(z_{i}+u_{i}\right)\right\}$. We denote by $\mu: \mathcal{D}_{c} \rightarrow$ $X_{\mathcal{D}}$ projection on $X_{\mathcal{D}}$, and by $\kappa: \mathcal{D}_{c} \rightarrow \mathfrak{z}_{\mathcal{D}}$ the projection on $\mathfrak{z}_{\mathcal{D}}$, so $\kappa\left(x, z_{1}, \ldots, z_{n}\right)=$ $\left(z_{1}, \ldots, z_{n}\right)$.

Remark 3.15. Recall the open subset $X_{\mathcal{D}, \text { gen }}$ of $X_{\mathcal{D}}$ defined in the introduction, and its preimage $\hat{\mathfrak{g}}_{\mathcal{D}, \text { gen }}=\mu^{-1}\left(X_{\mathcal{D}, \text { gen }}\right)$. In Remark[5.19, in the special case when all $D_{i}$ are regular semisimple, we identify the cover $\hat{\mathfrak{g}}_{\mathcal{D} \text {,gen }} \rightarrow X_{\mathcal{D} \text {,gen }}$ with the cover $M_{\Omega}(n, \mathfrak{e}) \rightarrow M_{\Omega}(n)$ that plays a key role in [KW06b]. In Theorem 5.18, we show that $\hat{\mathfrak{g}}_{\mathcal{D}}$ and $X_{\mathcal{D}}$ are connected.

\section{Poisson GeOmetry of $\dot{\mathfrak{g}}_{\mathcal{D}}$}

In this section, we use standard results from Poisson geometry to construct and compute a Poisson structure $\pi_{\mathcal{D}}$ on $\dot{\mathfrak{g}}_{\mathcal{D}}$.

4.1. Recollections from Poisson geometry. Let $(M, \pi)$ be a Poisson manifold. Thus, $\pi$ is a global section of $\wedge^{2}(T M)$, and if $f, g$ are functions on $M$, their Poisson bracket defined by $\{f, g\}:=\pi(d f, d g)$ makes the ring of functions on $M$ into a Poisson Lie algebra. Let $\tilde{\pi}: T_{x}^{*}(M) \rightarrow T_{x}(M)$ be the anchor map, defined by $\tilde{\pi}(\alpha)(\beta)=\pi(\alpha, \beta)$ if $\alpha, \beta$ are cotangent vectors at $x \in M$. For $f \in \mathbb{C}[M]$, we let $\xi_{f}:=\widetilde{\pi}(d f)$ be the Hamiltonian vector field of $f$. For $f, g \in \mathbb{C}[M],\left[\xi_{f}, \xi_{g}\right]=\xi_{\{f, g\}}$. If $N \subset M$ is a submanifold of $M$, its characteristic distribution is $\widetilde{\pi}\left(T_{N}^{*}(M)\right)$, where $T_{N}^{*}(M)$ is the conormal bundle to $N$ in $M$.

Definition 4.1. A submanifold $N$ of $M$ is called coisotropic with respect to $\pi$ if its characteristic distribution $\widetilde{\pi}\left(T_{N}^{*}(M)\right) \subset T N$.

Given two Poisson manifolds $\left(M, \pi_{M}\right)$ and $\left(R, \pi_{R}\right)$ a smooth map $\phi: M \rightarrow R$ is Poisson if $\phi_{*} \pi_{M}=\pi_{R}$.

Proposition 4.2. Let $\phi:\left(M, \pi_{M}\right) \rightarrow\left(R, \pi_{R}\right)$ be a surjective Poisson submersion between Poisson manifolds. Assume that

1) $Q$ is a coisotropic submanifold of $\left(M, \pi_{M}\right)$;

2) The characteristic distribution of $\pi_{M}$ on $Q$ is a subspace of the distribution defined by the tangent spaces to the fibers of $\phi$;

3) $\phi(Q)$ is a smooth submanifold of $R$;

Then $\phi(Q)$ is a Poisson submanifold of $\left(R, \pi_{R}\right)$.

We remark that this Proposition is a mild generalization of Proposition 6.7 from [EL07], and the proof given in [EL07] also works for our result here. 
4.2. The Poisson structure on $\dot{D}(\mathfrak{l}, x)$. A symplectic form $\omega$ on $M$ induces an identification $\widetilde{\omega}: T(M) \rightarrow T^{*}(M)$ given by $\widetilde{\omega}(\xi)(\eta)=\omega(\xi, \eta)$ for vector fields $\xi, \eta$ on $M$. We consider the bivector $\pi=\pi_{\omega}$ such that the second exterior power of $\widetilde{\omega}$ maps $\pi$ to $\omega$. Then $(M, \pi)$ is Poisson (see Section 1.2 of [CG97]).

We apply this construction to the case where $M=T^{*} K$ is the cotangent bundle of a reductive group $K$ with invariant nondegenerate bilinear form $\langle$,$\rangle on its Lie algebra \mathfrak{k}$. We identify $T^{*} K=K \times \mathfrak{k}^{*}=K \times \mathfrak{k}$, using left-invariant forms in the first identification and $\langle$,$\rangle for the second identification. Fix a point (g, \alpha) \in M=K \times \mathfrak{k}$.

Definition 4.3. (1) For $X \in \mathfrak{k}$, let $\xi_{X}$ be the tangent vector at $(g, \alpha)$ to the curve $t \mapsto$ $(g \exp t X, \alpha)$ at $t=0$;

(2) For $\beta \in \mathfrak{k}$, let $\eta_{\beta}$ be the tangent vector at $(g, \alpha)$ to the curve $t \mapsto(g, \alpha+t \beta)$ at $t=0$.

We identify $\mathfrak{k}+\mathfrak{k} \cong T_{(g, \alpha)}(M)$ via the map $(X, \beta) \mapsto\left(\xi_{X}, \eta_{\beta}\right)$. We identify the dual space $T_{(g, \alpha)}^{*}(M)=\mathfrak{k}+\mathfrak{k}$ using the form $\langle$,$\rangle . Using these identifications, a symplectic form$ $\omega_{c l}$ on $K \times \mathfrak{k}$ is given by the formula (cf. p. 497 of [KKS78]):

$$
\omega_{c l(g, \alpha)}\left(\left(X_{1}, \beta_{1}\right),\left(X_{2}, \beta_{2}\right)\right)=-<\beta_{1}, X_{2}>+<\beta_{2}, X_{1}>+<\alpha,\left[X_{1}, X_{2}\right]>.
$$

Up to sign, $\omega_{c l}$ coincides with the canonical symplectic form on the cotangent bundle from Section 1.1 of [CG97]. We denote by $\pi_{c l}=\pi_{\omega_{c l}}$ the induced Poisson bivector on $T^{*} K$. Then the anchor map of $\pi_{c l}$ is given by the formula:

$$
\widetilde{\pi_{c l}(g, \alpha)}(\gamma, Y)=(-Y, \gamma+[Y, \alpha]), \gamma, Y \in \mathfrak{k} .
$$

Recall the notation of Section 3.1, so $\mathfrak{p}$ is a parabolic subalgebra of $\mathfrak{k}$ with Levi decomposition $\mathfrak{p}=\mathfrak{l}+\mathfrak{u}$, and $\mathfrak{z}_{\text {gen }}$ is the generic part of the center $\mathfrak{z}$ of $\mathfrak{l}$.

Let $Q=K \times(z+L \cdot x+\mathfrak{u})$, where $z \in \mathfrak{z}_{\text {gen }}$ and $x \in \mathfrak{l}$ is nilpotent. Note that $P$ acts on $Q$ diagonally by $p \cdot(g, \alpha)=\left(g p^{-1}, \operatorname{Ad}(p) \alpha\right)$, for $p \in P,(g, \alpha) \in Q$.

Lemma 4.4. The subvariety $Q$ is a coisotropic subvariety of $M=K \times \mathfrak{k}$. Further, the characteristic distribution is tangent to the diagonal P-action on $Q$.

Proof. Let $(g, \alpha) \in Q$ and let $\alpha=z+y+u$, with $y \in L \cdot x$ and $u \in \mathfrak{u}$. Using the above identification $T_{(g, \alpha)}(M)=\mathfrak{k}+\mathfrak{k}, T_{(g, \alpha)}(Q)=(\mathfrak{k},[\mathfrak{l}, y]+\mathfrak{u})$, so that $\left(T_{Q}^{*}(M)\right)_{(g, \alpha)}=$ $\left(0,([\mathfrak{l}, y]+\mathfrak{u})^{\perp}\right)$. Note that

$$
([\mathfrak{l}, y]+\mathfrak{u})^{\perp}=[\mathfrak{l}, y]^{\perp} \cap \mathfrak{u}^{\perp}=\left(\mathfrak{u}^{-}+\mathfrak{z} \mathfrak{l}(y)+\mathfrak{u}\right) \cap \mathfrak{p}=\mathfrak{z} \mathfrak{l}(y)+\mathfrak{u},
$$

so that

$$
\left(T_{Q}^{*}(M)\right)_{(g, \alpha)}=(0, \mathfrak{z} \mathfrak{l}(y)+\mathfrak{u}) .
$$

Applying (4.1), we compute the characteristic distribution

$$
\widetilde{\pi_{c l}}\left(T_{Q}^{*}(M)\right)_{(g, \alpha)}=\{(-Y,[Y, \alpha]): Y \in \mathfrak{z} \mathfrak{l}(y)+\mathfrak{u}\} .
$$


It follows that the characteristic distribution is in the tangent space to the diagonal $P$ action. In particular, $Q$ is coisotropic.

\section{Q.E.D.}

Note that the diagonal $P$-action on $K \times \mathfrak{k}$ preserves the Poisson structure $\pi_{c l}$. Hence, if we consider the projection map pr: $K \times \mathfrak{k} \rightarrow K \times_{P} \mathfrak{k}$, then $\pi:=\operatorname{pr}_{*} \pi_{c l}$ is a well-defined Poisson structure on $K \times{ }_{P} \mathfrak{k}$. Since $\alpha: K \times_{P} \mathfrak{k} \rightarrow K / P \times \mathfrak{k}$ is an isomorphism, it follows that $\Pi:=\alpha_{*} \pi$ is a well-defined Poisson structure on $K / P \times \mathfrak{k}$. Further, if we let $\phi=\alpha \circ \mathrm{pr}$ so $\phi(g, \alpha)=(g P, \operatorname{Ad}(g) \alpha)$, then $\phi:\left(K \times \mathfrak{k}, \pi_{c l}\right) \rightarrow(K / P \times \mathfrak{k}, \Pi)$ is a Poisson morphism.

Proposition 4.5. Let $D(\mathfrak{l}, x)$ be a decomposition class. Then the variety $\dot{D}(\mathfrak{l}, x)$ is a Poisson subvariety of $\left(K \times_{P} \mathfrak{k}, \pi\right)$. The morphism $\mu: \dot{D}(\mathfrak{l}, x) \rightarrow \mathfrak{k}$ defined by $p(g, v)=$ $\operatorname{Ad}(g) v$ is Poisson.

Proof. Choose $z \in \mathfrak{z}_{\text {gen }}$. We apply Proposition 4.2 with $M=K \times \mathfrak{k}, Q=K \times(z+L \cdot x+\mathfrak{u})$ and $R=K \times{ }_{P} \mathfrak{k}$, and the quotient morphism $\phi: M \rightarrow R$ defined above.

By Lemma 4.4 and Proposition 4.2, $K \times_{P}(z+L \cdot x+\mathfrak{u})$ is a Poisson subvariety of $(R, \pi)$. We show that that $\pi$ is nondegenerate on $K \times_{P}(z+L \cdot x+\mathfrak{u})$. Note that the morphism $\mu: R \rightarrow \mathfrak{k}$ is Poisson by Lemma 1.4.2 and Proposition 1.4.10 of [CG97], so its restriction $\mu: K \times_{P}(z+L \cdot x+\mathfrak{u}) \rightarrow K \cdot(z+x)$ is a Poisson covering by Proposition 3.1 . Since $K \cdot(z+x)$ has nondegenerate Poisson structure, it follows that $K \times_{P}(z+L \cdot x+\mathfrak{u})$ is symplectic. Thus, $\dot{D}(\mathfrak{l}, x)$ is a union of symplectic leaves, and hence Poisson.

\section{Q.E.D.}

Remark 4.6. The symplectic structure on $\dot{D}(\mathfrak{l}, x)$ can also be realized by symplectic reduction (see [KKS78] and [CG97]).

Since each $\dot{D}\left(\mathfrak{l}_{i}, u_{i}\right)$ has Poisson structure $\pi_{i}$, the product $\dot{\mathfrak{g}}_{\mathcal{D}}=\prod_{i=1}^{n} \dot{D}\left(\mathfrak{l}_{i}, u_{i}\right)$ inherits a product Poisson structure $\pi_{\mathcal{D}}$. Further, the product $\tilde{\mathfrak{g}}_{\mathcal{D}}:=\prod_{i=1}^{n} \tilde{\mathfrak{g}}_{i}$ inherits a product Poisson structure $\Pi_{\mathcal{D}}$, and the product morphism $\alpha:\left(\dot{\mathfrak{g}}_{\mathcal{D}}, \pi_{\mathcal{D}}\right) \rightarrow\left(\tilde{\mathfrak{g}}_{\mathcal{D}}, \Pi_{\mathcal{D}}\right)$ from Equation (3.2) is Poisson.

4.3. Computation of the anchor map. In this section, we compute the anchor map on $K / P \times \mathfrak{k}$ for $\Pi$.

Since $\phi:\left(K \times \mathfrak{k}, \pi_{c l}\right) \rightarrow(K / P \times \mathfrak{k}, \Pi)$ is Poisson, it follows easily that $\widetilde{\Pi}_{(g P, \operatorname{Ad}(g) \alpha)}=$ $\phi_{(g, \alpha), *} \circ \widetilde{\pi_{c l}(g, \alpha)} \circ \phi_{(g, \alpha)}^{*}$. We factor $\phi=(p, \mu)$, where $p(g, \alpha)=g P$ and $\mu(g, \alpha)=\operatorname{Ad}(g) \alpha$. As in the last section, we identify $T_{(g, \alpha)}(K \times \mathfrak{k})=\mathfrak{k}+\mathfrak{k}$. We further identify

$$
\mathfrak{k} / \mathfrak{p}+\mathfrak{k} \cong T_{(g P, \beta)}(K / P \times \mathfrak{k}) ;(X+\mathfrak{p}, \gamma) \mapsto\left(\bar{\xi}_{X}, \eta_{\gamma}\right)
$$


where $\bar{\xi}_{X}$ is the tangent vector to the curve $t \mapsto(g \exp (t X) P, \beta)$ at $t=0$, and $\eta_{\gamma}$ is the tangent vector to the curve $(g P, \beta+t \gamma)$ at $t=0$. We identify $T_{(g P, \beta)}^{*}(K / P \times \mathfrak{k})=\mathfrak{u}+\mathfrak{k}$, using the identification $\mathfrak{u}=\mathfrak{p}^{\perp}$.

It is routine to check that if $(X, \beta) \in \mathfrak{k}+\mathfrak{k}$ and $\gamma \in \mathfrak{k}=T_{\operatorname{Ad}(g) \nu}^{*} \mathfrak{k}$, then the differential and codifferential are computed by

$$
\mu_{(g, \nu), *}(X, \beta)=\operatorname{Ad}(g)([X, \nu]+\beta), \quad \mu_{(g, \nu)}^{*}(\gamma)=\left(\left[\nu, \operatorname{Ad}\left(g^{-1}\right) \gamma\right], \operatorname{Ad}\left(g^{-1}\right) \gamma\right) .
$$

It follows that the differential

$$
\phi_{(g, \nu), *}(X, \beta)=(X+\mathfrak{p}, \operatorname{Ad}(g)([X, \nu]+\beta)),
$$

and hence the codifferential

$$
\phi_{(g, \nu)}^{*}(\lambda, \gamma)=\left(\lambda+\left[\nu, \operatorname{Ad}\left(g^{-1}\right) \gamma\right], \operatorname{Ad}\left(g^{-1}\right) \gamma\right), \lambda \in \mathfrak{u}, \gamma \in \mathfrak{k}
$$

Thus for $\lambda \in \mathfrak{u}$,

$$
\widetilde{\Pi}_{(g P, \operatorname{Ad}(g) \zeta)}(\lambda, 0)=\phi_{(g, \zeta), *} \widetilde{\pi_{c l}(g, \zeta)}(\lambda, 0)=\phi_{(g, \zeta), *}(0, \lambda)=(0, \operatorname{Ad}(g) \lambda),
$$

using Equations (4.6), (4.7), and (4.1). Further, using the same equations, we obtain

$$
\begin{aligned}
\widetilde{\Pi}_{(g P, \operatorname{Ad}(g) \zeta)}(0, \gamma) & =\phi_{(g, \zeta), *} \widetilde{\pi_{c l}(g, \zeta)}\left(\left[\zeta, \operatorname{Ad}\left(g^{-1}\right) \gamma\right], \operatorname{Ad}\left(g^{-1}\right) \gamma\right) \\
& =\phi_{(g, \zeta), *}\left(-\operatorname{Ad}\left(g^{-1}\right) \gamma, 0\right)=\left(-\operatorname{Ad}\left(g^{-1}\right) \gamma+\mathfrak{p},[\operatorname{Ad}(g) \zeta, \gamma]\right) .
\end{aligned}
$$

It follows that

$$
\widetilde{\Pi}_{(g P, \operatorname{Ad}(g) \zeta)}(\lambda, \gamma)=\left(-\operatorname{Ad}\left(g^{-1}\right) \gamma+\mathfrak{p},[\operatorname{Ad}(g) \zeta, \gamma]+\operatorname{Ad}(g) \lambda\right) .
$$

Remark 4.7. We can instead identify $\mathfrak{k} / \operatorname{Ad}(g) \mathfrak{p} \cong T_{g P}(K / P)$ by letting $X+\operatorname{Ad}(g) \mathfrak{p}$ be the tangent vector to $t \mapsto \exp (t X) g P$ at $t=0$, and use the corresponding identification $\operatorname{Ad}(g) \mathfrak{u} \cong T_{g P}^{*}(K / P)$. When we use these identifications, we obtain the formula for the anchor map

$$
\widetilde{\Pi}_{(g P, \operatorname{Ad}(g) \zeta)}(\lambda, \gamma)=(-\gamma+\operatorname{Ad}(g) \mathfrak{p},[\operatorname{Ad}(g) \zeta, \gamma]+\lambda) .
$$

With these identifications, it is clear that the anchor map does not depend on the representative chosen for the coset $g P$.

\section{Lifting of Gelfand-Zeitlin Fields TO $\hat{\mathfrak{g}}_{\mathcal{D}}$ AND ALGEBRAiC INTEGRABiLity}

In this section, let $\mathcal{D}=\left(D_{1}, \ldots, D_{n}\right)$ be regular decomposition data with $D_{i}=D\left(\mathfrak{l}_{i}, u_{i}\right)$. We introduce a collection of $\left(\begin{array}{l}n \\ 2\end{array}\right)$ functions $\hat{J}$ on $\dot{\mathfrak{g}}_{\mathcal{D}}$ and compute their Hamiltonian vector fields on $\hat{\mathfrak{g}}_{\mathcal{D}}$. More precisely, if $D_{i}=D\left(\mathfrak{l}_{i}, u_{i}\right)$, then we define $r_{i}=\operatorname{dim}\left(\mathfrak{z}_{i}\right)$ functions $q_{i, j}$ on $\dot{\mathfrak{g}}_{\mathcal{D}}$ using $\mathfrak{z}_{i}$, and we define $s_{i}=i-r_{i}$ functons $p_{i, k}$ on $\dot{\mathfrak{g}}_{\mathcal{D}}$ using the semisimple part $\left[\mathfrak{l}_{i}, \mathfrak{l}_{i}\right]$ of $\mathfrak{l}_{i}$. The functions $\hat{J}_{i}=\left\{q_{i, j}, p_{i, k}: j=1, \ldots, r_{i}, k=1, \ldots, s_{i}\right\}$ give $i$ functions on $\hat{\mathfrak{g}}_{\mathcal{D}}$, and we define $\hat{J}=\bigcup_{i=1}^{n-1} \hat{J}_{i}$. We let $\hat{\mathfrak{a}}$ be the linear span of $\left\{\xi_{f}: f \in \hat{J}\right\}$. We further show that $\hat{\mathfrak{a}}$ is abelian, and the vector fields in $\hat{\mathfrak{a}}$ lift the vector fields in $\mathfrak{a}$ on $X_{\mathcal{D}}$ to $\hat{\mathfrak{g}}_{\mathcal{D}}$ and are algebraically integrable on the $\Sigma_{\mathcal{D}}$-cover $\hat{\mathfrak{g}}_{\mathcal{D}}$ of $X_{\mathcal{D}}$. 
Throughout this section, we denote by $y=\left(g_{1}, y^{1}, \ldots, g_{n}, y^{n}\right)$ an element of $\hat{\mathfrak{g}}_{\mathcal{D}}$.

5.1. Functions associated to the center. In this section, we introduce functions associated to the center of the Levi factor $\mathfrak{l}_{i}$.

For each $i, 1 \leq i \leq n$, let $D_{i}$ be a decomposition class of $\mathfrak{g}_{i}$. As in Remark 3.5, the decomposition class $D_{i}$ determines a partition $i=i_{1}+\cdots+i_{r_{i}}$, normalized so $i_{k} \geq i_{j}$ when $k \leq j$. Let $\mathfrak{l}_{i}$ be the standard Levi subalgebra with diagonal blocks of sizes $\left(i_{1}, \ldots, i_{r_{i}}\right)$ and let $L_{i} \cdot u_{i}$ be the regular nilpotent orbit. Then $\mathfrak{z}_{i}=\sum_{k=1}^{r_{i}} \mathbb{C} \cdot \mathrm{id}_{i_{k}}$, where $\mathrm{id}_{i_{k}}$ is the identity matrix in the kth block, and 0 outside the kth block. Consider the invariant symmetric form on $\mathfrak{g}$ given by $\langle X, Y\rangle=\operatorname{Tr}(X \cdot Y)$. The restriction of $\langle\cdot, \cdot\rangle$ to $\mathfrak{g}_{i}$ is nondegenerate and $\mathfrak{g}_{i}{ }^{\perp}$ is spanned by elementary matrices not in $\mathfrak{g}_{i}$. In particular, $\mathfrak{g}$ is a direct sum

$$
\mathfrak{g}=\mathfrak{g}_{i} \oplus \mathfrak{g}_{i}^{\perp} .
$$

Remark 5.1. It is easy to see that both components of the decomposition in (5.1) are $\operatorname{Ad}\left(G_{i}\right)$ and hence $\operatorname{ad}\left(\mathfrak{g}_{i}\right)$ invariant.

Further, the restriction of the form is nondegenerate on $\mathfrak{l}_{i}$ and $\mathfrak{z}_{i}$. Let $\left\{z_{i, 1}, \ldots, z_{i, r_{i}}\right\}$ be the dual basis in $\mathfrak{z}_{i}$ to the basis $\left\{\mathrm{id}_{i_{1}}, \ldots, \mathrm{id}_{i_{r_{i}}}\right\}$ of $\mathfrak{z}_{i}$. Then $<z_{i, j}, z>=\lambda_{j}$ where $z=\sum_{i=1}^{r_{i}} \lambda_{k} \operatorname{id}_{i_{k}}$, so that pairing with $z_{i, j}$ computes the $j t h$ eigenvalue of $z$. It is easy to check that

$$
z_{i, j}=\frac{\mathrm{id}_{i_{j}}}{i_{j}}
$$

For $j=1, \ldots, r_{i}$, we define a function $q_{i, j}$ on $\dot{\mathfrak{g}}_{\mathcal{D}}=\prod_{k=1}^{n} G_{k} \times_{L_{k}}\left(\mathfrak{z}_{k, \text { gen }}+L_{k} \cdot u_{k}\right)$ by the formula:

$$
q_{i, j}\left(g_{1}, y^{1}, \ldots, g_{n}, y^{n}\right)=\sum_{s=i+1}^{n}<\operatorname{Ad}\left(g_{s}\right) y^{s}, \operatorname{Ad}\left(g_{i}\right) z_{i, j}>,
$$

where $g_{k} \in G_{k}$ and $y^{k} \in \mathfrak{z}_{k, \text { gen }}+L_{k} \cdot u_{k}$ for $k=1, \ldots, n$. It is routine to check that $q_{i, j}$ is a well-defined regular function on $\dot{\mathfrak{g}}_{\mathcal{D}}$.

For $i=1, \ldots, n$, we fix a parabolic subgroup $P_{i}$ containing $L_{i}$, and recall that $\tilde{\mathfrak{g}}_{\mathcal{D}}=$ $\prod_{i=1}^{n}\left(G_{i} / P_{i} \times \mathfrak{g}_{i}\right)$

At a point $v=\left(g_{1} P_{1}, x^{1}, \ldots, g_{n} P_{n}, x^{n}\right)$ of $\tilde{\mathfrak{g}}_{\mathcal{D}}$, we identify the tangent space $T_{v}\left(\tilde{\mathfrak{g}}_{\mathcal{D}}\right)=$ $\oplus_{i=1}^{n}\left(\mathfrak{g}_{i} / \mathfrak{p}_{i}+\mathfrak{g}_{i}\right)$ using the product of the identifications of Equation (4.4). Similarly, we identify $T_{v}^{*}\left(\tilde{\mathfrak{g}}_{\mathcal{D}}\right)=\oplus_{i=1}^{n}\left(\mathfrak{u}_{i}+\mathfrak{g}_{i}\right)$. For each $j=1, \ldots, r_{i}$, we define a covector $\tilde{\lambda}_{i, j} \in$ $\oplus_{i=1}^{n}\left(\mathfrak{u}_{i}+\mathfrak{g}_{i}\right)$ by setting 


$$
\tilde{\lambda}_{i, j}=(0,0, \ldots, \underbrace{0,0}_{\text {ith }}, \underbrace{0, \operatorname{Ad}\left(g_{i}\right) z_{i, j}}_{\text {i }+1 \text { st }}, \ldots, \underbrace{0, \operatorname{Ad}\left(g_{i}\right) z_{i, j}}_{\text {nth }}),
$$

so that the first $i$ pairs are $(0,0) \in \mathfrak{u}_{k} \oplus \mathfrak{g}_{k}, 1 \leq k \leq i$, and the last $(n-i)$ pairs are $\left(0, \operatorname{Ad}\left(g_{i}\right) z_{i, j}\right) \in \mathfrak{u}_{k} \oplus \mathfrak{g}_{k}, i+1 \leq k \leq n$.

Recall the morphism $\alpha: \dot{\mathfrak{g}}_{\mathcal{D}} \rightarrow \tilde{\mathfrak{g}}_{\mathcal{D}}$ from Equation (3.2).

Proposition 5.2. Let $y \in \hat{\mathfrak{g}}_{\mathcal{D}}$. Then

$$
\alpha_{y}^{*}\left(\tilde{\lambda}_{i, j}\right)=d q_{i, j}(y) .
$$

Proof. It suffices to verify that

$$
\tilde{\lambda}_{i, j}\left(\alpha_{y, *}(\chi)\right)=d q_{i, j}(y)(\chi),
$$

for each tangent vector $\chi$ in a generating set of tangent vectors of $T_{y}\left(\dot{\mathfrak{g}}_{\mathcal{D}}\right)$.

For $B_{k} \in \mathfrak{g}_{k}$, we let $\xi_{B_{k}} \in T_{y}\left(\dot{\mathfrak{g}}_{\mathcal{D}}\right)$ be the tangent vector at $t=0$ to the curve

$$
t \mapsto\left(g_{1}, y^{1}, \ldots, g_{k} \exp \left(t B_{k}\right), y^{k}, \ldots, g_{n}, y^{n}\right),
$$

constant in all directions except the $\left(g_{k}, y^{k}\right)$ direction. For $C_{k} \in \mathfrak{z}_{k}$, we let $\eta_{C_{k}} \in T_{y}\left(\dot{\mathfrak{g}}_{\mathcal{D}}\right)$ be the tangent vector at $t=0$ to the curve

$$
t \mapsto\left(g_{1}, y^{1}, \ldots, g_{k}, y^{k}+t C_{k}, \ldots, g_{n}, y^{n}\right),
$$

constant in all directions except the $\left(g_{k}, y^{k}\right)$ direction. For $k=1, \ldots, n$, the tangent vectors $\xi_{B_{k}}, \eta_{C_{k}}$ generate $T_{y}\left(\dot{\mathfrak{g}}_{\mathcal{D}}\right)$.

For $k<i, d q_{i, j}\left(\xi_{B_{k}}\right)=0$ since $q_{i, j}$ is constant along the corresponding flows. For $k \leq i$, $d q_{i, j}\left(\eta_{C_{k}}\right)=0$ for the same reason.

For the case $k=i$,

$$
\begin{aligned}
d q_{i, j}(y)\left(\xi_{B_{i}}\right) & =\left.\frac{d}{d t}\right|_{t=0} \sum_{s=i+1}^{n}<\operatorname{Ad}\left(g_{s}\right) y^{s}, \operatorname{Ad}\left(g_{i} \exp \left(t B_{i}\right)\right) z_{i, j}> \\
& =\sum_{s=i+1}^{n}<\operatorname{Ad}\left(g_{s}\right) y^{s}, \operatorname{Ad}\left(g_{i}\right)\left[B_{i}, z_{i, j}\right]>.
\end{aligned}
$$

We claim that $d q_{i, j}(y)\left(\xi_{B_{i}}\right)=0$. For this, it suffices to prove that for each $s>i$, $<\operatorname{Ad}\left(g_{s}\right) y^{s}, \operatorname{Ad}\left(g_{i}\right)\left[B_{i}, z_{i, j}\right]>=<\left[z_{i, j}, \operatorname{Ad}\left(g_{i}^{-1} g_{s}\right) y^{s}\right], B_{i}>=0$. By Equation (5.1),

$$
<\left[z_{i, j}, \operatorname{Ad}\left(g_{i}^{-1} g_{s}\right) y^{s}\right], B_{i}>=<\left[z_{i, j}, \operatorname{Ad}\left(g_{i}^{-1} g_{s}\right) y^{s}\right]_{i}, B_{i}>
$$


Since $y \in \hat{\mathfrak{g}}_{\mathcal{D}},\left(\operatorname{Ad}\left(g_{s}\right) y_{s}\right)_{i}=\operatorname{Ad}\left(g_{i}\right) y_{i}$ by Remark 3.11. Since $z_{i, j} \in \mathfrak{g}_{i}$, it follows from Remark 5.1 that we may rewrite (5.7) as

$$
<\left[z_{i, j}, \operatorname{Ad}\left(g_{i}^{-1}\right)\left(\operatorname{Ad}\left(g_{s}\right) y^{s}\right)_{i}\right], B_{i}>=<\left[z_{i, j}, y^{i}\right], B_{i}>=0
$$

since $y^{i} \in \mathfrak{l}_{i}$.

For $k>i$,

$$
d q_{i, j}(y)\left(\xi_{B_{k}}\right)=\left.\frac{d}{d t}\right|_{t=0}<\operatorname{Ad}\left(g_{k} \exp \left(t B_{k}\right)\right) y^{k}, \operatorname{Ad}\left(g_{i}\right) z_{i, j}>=<\operatorname{Ad}\left(g_{k}\right)\left[B_{k}, y^{k}\right], \operatorname{Ad}\left(g_{i}\right) z_{i, j}>.
$$

It is now straightforward to verify Equation (5.4) for $\chi=\xi_{B_{k}}$ using the formula for $\tilde{\lambda}_{i, j}$. An easier version of the above computation implies Equation (5.4) for $\chi=\eta_{C_{k}}$. This completes the proof.

\section{Q.E.D.}

We now compute the Hamiltonian vector fields $\xi_{q_{i, j}}$ of the functions $q_{i, j}$ on $\hat{\mathfrak{g}}_{\mathcal{D}}$.

Proposition 5.3. For the Poisson structure $\pi_{\mathcal{D}}$ on $\dot{\mathfrak{g}}_{\mathcal{D}}$ and a point $y=\left(g_{1}, y^{1}, \ldots, g_{n}, y^{n}\right)$ of $\hat{\mathfrak{g}}_{\mathcal{D}}$, the pushforward $\alpha_{y, *} \xi_{q_{i, j}}$ has $\left(\mathfrak{g}_{k} / \mathfrak{p}_{k}, \mathfrak{g}_{k}\right)$-component $(0,0)$ for $k \leq i$ and has $\left(\mathfrak{g}_{k} / \mathfrak{p}_{k}, \mathfrak{g}_{k}\right)$ component

$$
\left(-\operatorname{Ad}\left(g_{k}^{-1} g_{i}\right) z_{i, j}+\mathfrak{p}_{k},\left[\operatorname{Ad}\left(g_{k}\right) y^{k}, \operatorname{Ad}\left(g_{i}\right) z_{i, j}\right]\right)
$$

for $k>i$.

Proof. By Proposition 5.2 ,

$$
\alpha_{y, *}\left(\xi_{q_{i, j}}\right)=\alpha_{y, *} \widetilde{\pi_{\mathcal{D}}} \alpha_{y}^{*}\left(\tilde{\lambda}_{i, j}\right) .
$$

Since the embedding $\alpha:\left(\dot{\mathfrak{g}}_{\mathcal{D}}, \pi_{\mathcal{D}}\right) \rightarrow\left(\tilde{\mathfrak{g}}_{\mathcal{D}}, \Pi_{\mathcal{D}}\right)$ is Poisson (see Section 4.2),

$$
\alpha_{y, *} \widetilde{\pi_{\mathcal{D}}} \alpha_{y}^{*}\left(\tilde{\lambda}_{i, j}\right)=\widetilde{\Pi_{\mathcal{D}} \alpha(y)}\left(\tilde{\lambda}_{i, j}\right)
$$

The Proposition now follows easily from Equation (4.10).

\section{Q.E.D.}

We now define a curve $\theta_{i, j}(y, t)$ that integrates the vector field $\xi_{q_{i, j}}$ on $\hat{\mathfrak{g}}_{\mathcal{D}}$. For $y=$ $\left(g_{1}, y^{1}, \ldots, g_{n}, y^{n}\right) \in \dot{\mathfrak{g}}_{\mathcal{D}}$, we let

$$
h_{i, j}(y, t)=\exp \left(-t \operatorname{Ad}\left(g_{i}\right) z_{i, j}\right) .
$$

We define the curve $\theta_{i, j}(y, t)$ in $\dot{\mathfrak{g}}_{\mathcal{D}}$ by the equation

$$
t \mapsto\left(g_{1}, y^{1}, \ldots, g_{i}, y^{i}, h_{i, j}(y, t) g_{i+1}, y^{i+1}, \ldots, h_{i, j}(y, t) g_{n}, y^{n}\right)
$$


Proposition 5.4. The curve $\theta_{i, j}(y, t)$ is an integral curve for $\xi_{q_{i, j}}$ on $\hat{\mathfrak{g}}_{\mathcal{D}}$, and induces an algebraic action of $\mathbb{C}^{\times}$on $\hat{\mathfrak{g}}_{\mathcal{D}}$.

Proof. Suppose $y \in \hat{\mathfrak{g}}_{\mathcal{D}}$. Let $x^{i}=\operatorname{Ad}\left(g_{i}\right) y^{i}$ for $i=1, \ldots, n$. To show that $\theta_{i, j}(y, t) \in \hat{\mathfrak{g}}_{\mathcal{D}}$, we must verify that for $k>i$,

$$
\left(\operatorname{Ad}\left(h_{i, j}(y, t)\right) x^{n}\right)_{k}=\operatorname{Ad}\left(h_{i, j}(y, t)\right) x^{k}
$$

and for $k \leq i$,

$$
\left(\operatorname{Ad}\left(h_{i, j}(y, t)\right) x^{n}\right)_{k}=x^{k}
$$

and also verify that $\operatorname{Ad}\left(h_{i, j}(y, t)\right) x^{n}$ is strongly regular. For $k>i, h_{i, j}(y, t) \in G_{i} \subset G_{k}$, so by Remark 5.1 we may rewrite the left side of (5.9) as

$$
\operatorname{Ad}\left(h_{i, j}(y, t)\right)\left(x^{n}\right)_{k}=\operatorname{Ad}\left(h_{i, j}(y, t)\right) x^{k},
$$

since $\left(x^{n}\right)_{k}=x^{k}$ by Remark 3.11. Now, if $k \leq i$, then

$$
\left(\operatorname{Ad}\left(h_{i, j}(y, t)\right) x^{n}\right)_{k}=\left(\operatorname{Ad}\left(h_{i, j}(y, t)\right)\left(x^{n}\right)_{i}\right)_{k}=\left(\operatorname{Ad}\left(h_{i, j}(y, t)\right) x^{i}\right)_{k}=\left(x^{i}\right)_{k}=x^{k}
$$

since $h_{i, j}(y, t)$ centralizes $x^{i}$. To verify that $\operatorname{Ad}\left(h_{i, j}(y, t)\right) x^{n}$ is strongly regular, we use Equations (5.9) and (5.10). We first observe that $\left(\operatorname{Ad}\left(h_{i, j}(y, t)\right) x^{i}\right)_{k}$ is regular in $\mathfrak{g}_{k}$ by Equations (5.9) and (5.10) and Theorem 2.1 (3). For $k<i$, Equation (5.10) implies,

$$
\mathfrak{z}_{\mathfrak{g}_{k}}\left(\left(\operatorname{Ad}\left(h_{i, j}(y, t)\right) \cdot x^{n}\right)_{k}\right) \cap \mathfrak{z}_{\mathfrak{g}_{k+1}}\left(\left(\operatorname{Ad}\left(h_{i, j}(y, t)\right) \cdot x^{n}\right)_{k+1}\right)=\mathfrak{z}_{\mathfrak{g}_{k}}\left(x^{k}\right) \cap \mathfrak{z} \mathfrak{g}_{k+1}\left(x^{k+1}\right)=0,
$$

by Theorem 2.1 (3). For $k \geq i$, by (5.9),

$$
\begin{gathered}
\mathfrak{z}_{\mathfrak{g}_{k}}\left(\left(\operatorname{Ad}\left(h_{i, j}(y, t)\right) \cdot x^{n}\right)_{k}\right) \cap \mathfrak{z}_{\mathfrak{g}_{k+1}}\left(\left(\operatorname{Ad}\left(h_{i, j}(y, t)\right) \cdot x^{n}\right)_{k+1}\right) \\
=\operatorname{Ad}\left(h_{i, j}(y, t)\right)\left(\mathfrak{z} \mathfrak{g}_{k}\left(x^{k}\right) \cap \mathfrak{z} \mathfrak{g}_{k+1}\left(x^{k+1}\right)\right)=0 .
\end{gathered}
$$

Thus, by Theorem 2.1, $\operatorname{Ad}\left(h_{i, j}(y, t)\right) x^{n}$ is strongly regular and $\theta_{i, j}(y, t) \in \hat{\mathfrak{g}}_{\mathcal{D}}$ for $y \in \hat{\mathfrak{g}}_{\mathcal{D}}$.

By Proposition 5.3, it follows that $\frac{d}{d t} \theta_{i, j}(y, t)=\xi_{q_{i, j}}$. Consider the element $\tilde{h}_{i, j}(y, s)=$ $g_{i} A_{j}(s) g_{i}^{-1} \in G_{i}$, where $s \in \mathbb{C}^{\times}$and $A_{j}(s)$ is the diagonal matrix in $G_{i}$ with scalar matrix $s$ in the jth block of $\mathfrak{l}_{i}$ and 1 elsewhere on the diagonal. We define a curve $\tilde{\theta}_{i, j}(y, s)$ in $\dot{\mathfrak{g}}_{\mathcal{D}}$ using Equation (5.8) with $\tilde{h}_{i, j}(y, s)$ in place of $h_{i, j}(y, t)$. It follows from Equations (5.2) and (5.8) that $\tilde{\theta}_{i, j}(y, s)=\theta_{i, j}(y, t)$ when $s=\exp \left(-\frac{t}{i_{j}}\right)$, which completes the proof of the proposition.

Q.E.D. 
5.2. Functions associated to the semisimple part. Let $s_{i}=i-\operatorname{dim}\left(\mathfrak{z}_{i}\right)$. In this section, we find $s_{i}$ algebraically independent functions on $\dot{\mathfrak{g}}_{\mathcal{D}}$ and show their Hamiltonian vector fields integrate to an algebraic action on $\hat{\mathfrak{g}}_{\mathcal{D}}$.

Let $\mathfrak{l}_{i}$ be the standard Levi factor associated to the decomposition class $D_{i}$. We decompose $\mathfrak{l}_{i}=\mathfrak{s}_{i} \oplus \mathfrak{z}_{i}$, where $\mathfrak{s}_{i}=\left[\mathfrak{l}_{i}, \mathfrak{l}_{i}\right]$ is the derived algebra of $\mathfrak{l}_{i}$, and note that the form $\langle$, is nondegenerate on $\mathfrak{s}_{i}$.

For $x \in \mathfrak{s}_{i}$, and $f \in \mathbb{C}\left[\mathfrak{s}_{i}\right]$, we define the gradient $\nabla f(x) \in \mathfrak{s}_{i}$ by the property that $<\nabla f(x), u>=d f(x)(u)=\left.\frac{d}{d t}\right|_{t=0} f(x+t u)$, for $u \in \mathfrak{s}_{i}$. It follows from definitions that if $g \in L_{i}$ and $\operatorname{Ad}(g) f=f$, then

$$
\nabla f(\operatorname{Ad}(g) x)=\operatorname{Ad}(g)(\nabla f(x)) .
$$

Further, if $f \in \mathbb{C}\left[\mathfrak{s}_{i}\right]^{Z_{L_{i}}(x)}$, then

$$
[\nabla f(x), u]=0 \forall u \in \mathfrak{z}_{\mathfrak{s}_{i}}(x) .
$$

Lemma 5.5. Let $f_{1}, f_{2} \in \mathbb{C}\left[\mathfrak{s}_{i}\right]^{L_{i}}$. Then $\left[\nabla f_{1}(x), \nabla f_{2}(x)\right]=0$.

Proof. By Equation (5.12) with $u=x, \nabla f_{i}(x) \in \mathfrak{z}_{\mathfrak{s}_{i}}(x)$. The result follows immediately from another application of Equation (5.12) with $u=\nabla f_{i}(x)$.

Q.E.D.

Let now $f \in \mathbb{C}\left[\mathfrak{s}_{i}\right]^{L_{i}}$ be an invariant function, and define a regular function $\tilde{f}$ on $\dot{\mathfrak{g}}_{\mathcal{D}}$ by the formula:

$$
\tilde{f}\left(g_{1}, y^{1}, \ldots, g_{n}, y^{n}\right)=\sum_{s=i+1}^{n}<\operatorname{Ad}\left(g_{s}\right) y^{s}, \operatorname{Ad}\left(g_{i}\right) \nabla f\left(n^{i}\right)>
$$

where $y^{i}=s^{i}+n^{i}$ is the Jordan decomposition of $y^{i}$. By Equation (5.11), $\tilde{f}$ is a welldefined regular function on $\dot{\mathfrak{g}}_{\mathcal{D}}$.

Let $y=\left(g_{1}, y^{1}, \ldots, g_{n}, y^{n}\right) \in \hat{\mathfrak{g}}_{\mathcal{D}}$. Using the identification $T_{\alpha(y)}^{*}\left(\tilde{\mathfrak{g}}_{\mathcal{D}}\right)=\oplus\left(\mathfrak{u}_{i}+\mathfrak{g}_{i}\right)$, we regard the vector

$$
\psi=(0,0, \ldots, \underbrace{0,0}_{\text {ith }}, \underbrace{0, \operatorname{Ad}\left(g_{i}\right) \nabla f\left(n_{i}\right)}_{\text {i }+1 \text { st }}, \ldots, \underbrace{0, \operatorname{Ad}\left(g_{i}\right) \nabla f\left(n_{i}\right)}_{\text {nth }})
$$

as a cotangent vector at $\alpha(y) \in \tilde{\mathfrak{g}}_{\mathcal{D}}$.

Proposition 5.6. For $y \in \hat{\mathfrak{g}}_{\mathcal{D}}, \alpha_{y}^{*}(\psi)=d \tilde{f}(y)$. 
Proof. We must check that

$$
\psi\left(\alpha_{*, y}(\chi)\right)=d \tilde{f}(y)(\chi)
$$

for each tangent vector $\chi$ in a generating set of tangent vectors of $T_{y}\left(\dot{\mathfrak{g}}_{\mathcal{D}}\right)$. It suffices to check this assertion when $\chi$ is the evaluation of a vector field $\xi_{B_{k}}$ with $B_{k} \in \mathfrak{g}_{k}$ or the evaluation of a vector field $\eta_{C_{k}}$ with $C_{k} \in \mathfrak{z}_{k}$ at $y$ (see Equations (5.5) and (5.6)). If $k<i$, the assertion is trivial as both sides are 0 . If $k>i$, we compute

$$
d \tilde{f}(y)\left(\xi_{B_{k}}\right)=<\operatorname{Ad}\left(g_{k}\right)\left[B_{k}, y^{k}\right], \operatorname{Ad}\left(g_{i}\right) \nabla f\left(n^{i}\right)>=\psi\left(\alpha_{*, y} \xi_{B_{k}}\right) .
$$

If $k=i$, we compute

$$
d \tilde{f}(y)\left(\xi_{B_{i}}\right)=\left.\frac{d}{d t}\right|_{t=0} \sum_{s=i+1}^{n}<\operatorname{Ad}\left(g_{s}\right) y^{s}, \operatorname{Ad}\left(g_{i} \exp \left(t B_{i}\right)\right) \nabla f\left(n^{i}\right)>.
$$

Since $\exp \left(-t B_{i}\right) \in G_{i}, \nabla f\left(n^{i}\right) \in \mathfrak{g}_{i}$, and $\left(\operatorname{Ad}\left(g_{s}\right) y^{s}\right)_{i}=\operatorname{Ad}\left(g_{i}\right) y^{i}$

$$
\begin{aligned}
16)<\operatorname{Ad}\left(g_{s}\right) y^{s}, \operatorname{Ad}\left(g_{i} \exp \left(t B_{i}\right)\right) \nabla f\left(n^{i}\right)>=<\operatorname{Ad}\left(\exp \left(-t B_{i}\right) g_{i}^{-1} g_{s}\right) y^{s}, \nabla f\left(n^{i}\right)> \\
=<\left(\operatorname{Ad}\left(\exp \left(-t B_{i}\right) g_{i}^{-1} g_{s}\right) y^{s}\right)_{i}, \nabla f\left(n^{i}\right)>=<\operatorname{Ad}\left(\exp \left(-t B_{i}\right)\right) y^{i}, \nabla f\left(n^{i}\right)>.
\end{aligned}
$$

Hence, we may rewrite Equation (5.15) as

$$
d \tilde{f}(y)\left(\xi_{B_{i}}\right)=\left.\frac{d}{d t}\right|_{t=0} \sum_{s=i+1}^{n}<\operatorname{Ad}\left(\exp \left(-t B_{i}\right)\right) y^{i}, \nabla f\left(n^{i}\right)>
$$

But

$$
\left.\frac{d}{d t}\right|_{t=0}<\operatorname{Ad}\left(\exp \left(-t B_{i}\right)\right) y^{i}, \nabla f\left(n^{i}\right)>=<-B_{i},\left[y^{i}, \nabla f\left(n^{i}\right)\right]>=0
$$

using Equation (5.12), and Equation (5.14) follows easily for $\chi=\xi_{B_{k}}$. The verification of Equation (5.14) for $\chi=\eta_{C_{k}}$ is left to the reader.

\section{Q.E.D.}

We denote by $y^{i}=s^{i}+n^{i}$ the Jordan decomposition of $y^{i} \in D\left(\mathfrak{l}_{i}, u_{i}\right)$ for the remainder of the paper. We choose $s_{i}$ algebraically independent functions $\phi_{i, j} \in \mathbb{C}\left[\mathfrak{s}_{i}\right]^{L_{i}}$. For $j=$ $1, \ldots, s_{i}$, we define a regular function $p_{i, j}$ on $\dot{\mathfrak{g}}_{\mathcal{D}}$ by $p_{i, j}=\tilde{\phi}_{i, j}$, using Equation (5.13) with $f=\phi_{i, j}$. 
Remark 5.7. The set $\left\{\nabla \phi_{i, j}\left(n^{i}\right): j=1, \ldots, s_{i}\right\}$ is a basis of $\mathfrak{z}_{\mathfrak{s}_{i}}\left(n^{i}\right)$. First note that $\nabla \phi_{i, j}\left(n^{i}\right) \in \mathfrak{z}_{\mathfrak{s}_{i}}\left(n^{i}\right)$ by Equation (5.12). Since $n^{i}$ is principal nilpotent in $\mathfrak{s}_{i}, \operatorname{dim} \mathfrak{z}_{\mathfrak{s}_{i}}\left(n^{i}\right)=$ $\operatorname{rank}\left(\mathfrak{s}_{i}\right)=s_{i}$, and the elements $\left\{\nabla \phi_{i, j}\left(n^{i}\right): j=1, \ldots, s_{i}\right\}$ are linearly independent by a well-known result of Kostant [Kos63], Theorem 9.

By Proposition 5.6, it follows that for $y \in \hat{\mathfrak{g}}_{\mathcal{D}}$,

$$
d p_{i, j}(y)=\alpha_{y}^{*}\left(\psi_{i, j}\right)
$$

where $\psi_{i, j}$ is the vector in $\oplus\left(\mathfrak{u}_{i}+\mathfrak{g}_{i}\right)$ given by

$$
\psi_{i, j}=(0,0, \ldots, \underbrace{0,0}_{\text {ith }}, \underbrace{0, \operatorname{Ad}\left(g_{i}\right) \nabla \phi_{i, j}\left(n^{i}\right)}_{\mathrm{i}+1 \mathrm{st}}, \ldots, \underbrace{0, \operatorname{Ad}\left(g_{i}\right) \nabla \phi_{i, j}\left(n^{i}\right)}_{\text {nth }}) .
$$

Proposition 5.8. For the Poisson structure $\pi_{\mathcal{D}}$ on $\dot{\mathfrak{g}}_{\mathcal{D}}$ and $y=\left(g_{1}, y^{1}, \ldots, g_{n}, y^{n}\right) \in \hat{\mathfrak{g}}_{\mathcal{D}}$, the kth component in $\left(\mathfrak{g}_{k} / \mathfrak{p}_{k}, \mathfrak{g}_{k}\right)$ of the pushforward $\alpha_{y, *} \xi_{p_{i, j}}$ is $(0,0)$ if $k \leq i$, and if $k>i$, it is

$$
\left(-\operatorname{Ad}\left(g_{k}^{-1} g_{i}\right) \nabla \phi_{i, j}\left(n^{i}\right)+\mathfrak{p}_{k},\left[\operatorname{Ad}\left(g_{k}\right) y^{k}, \operatorname{Ad}\left(g_{i}\right) \nabla \phi_{i, j}\left(n^{i}\right)\right]\right) .
$$

The proof is similar to the proof of Proposition 5.3, and we leave details to the reader.

We define

$$
A_{i, j}(y, t)=\exp \left(-t \operatorname{Ad}\left(g_{i}\right)\left(\nabla \phi_{i, j}\left(n^{i}\right)\right)\right)
$$

Since $n^{i}$ is principal nilpotent in $\mathfrak{s}_{i}, \nabla \phi_{i, j}\left(n^{i}\right) \in \mathfrak{z}_{\mathfrak{s}_{i}}\left(n^{i}\right)$ is nilpotent by a well-known result of Kostant (see Remark 35.1.4 in [TY05]). It follows that the morphism $t \mapsto$ $A_{i, j}(y, t)$ is algebraic.

For each $j=1, \ldots, s_{i}$, define a curve in $\dot{\mathfrak{g}}_{\mathcal{D}}$ by the formula

$$
\mu_{i, j}(y, t)=\left(g_{1}, y^{1}, \ldots, g_{i}, y^{i}, A_{i, j}(y, t) g_{i+1}, y^{i+1}, \ldots, A_{i, j}(y, t) g_{n}, y^{n}\right) .
$$

Proposition 5.9. The curve $\mu_{i, j}(y, t)$ is an integral curve for the Hamiltonian vector field $\xi_{p_{i, j}}$ on $\hat{\mathfrak{g}}_{\mathcal{D}}$, and induces an algebraic action of the additive group $\mathbb{C}$ on $\hat{\mathfrak{g}}_{\mathcal{D}}$.

The proof is similar to the proof of Proposition 5.4 and the details are left to the reader.

\subsection{Lift of the $A$-action to $\hat{\mathfrak{g}}_{\mathcal{D}}$. Let}

$$
\hat{J}_{i}=\left\{q_{i, j}: j=1, \ldots, r_{i}=\operatorname{dim}\left(\mathfrak{z}_{i}\right)\right\} \cup\left\{p_{i, k}: k=1, \ldots, s_{i}=i-r_{i}\right\},
$$

and let $\hat{J}=\bigcup_{i=1}^{n-1} \hat{J}_{i}$. 
Let

$$
\hat{\mathfrak{a}}_{i}:=\operatorname{span}\left\{\xi_{f}: f \in \hat{J}_{i}\right\} \subset \Gamma\left(\hat{\mathfrak{g}}_{\mathcal{D}}, T \hat{\mathfrak{g}}_{\mathcal{D}}\right), \quad \hat{\mathfrak{a}}:=\sum_{i=1}^{n-1} \hat{\mathfrak{a}}_{i} .
$$

Recall the étale covering $\mu: \hat{\mathfrak{g}}_{\mathcal{D}} \rightarrow X_{\mathcal{D}}$. In this section, we show that $\hat{\mathfrak{a}}$ is an abelian Lie algebra of dimension $\left(\begin{array}{l}n \\ 2\end{array}\right)$, and $\mu_{*} \hat{\mathfrak{a}}=\mathfrak{a}$, so that $\hat{\mathfrak{a}}$ lifts the action of $\mathfrak{a}$ to the covering $\hat{\mathfrak{g}}_{\mathcal{D}}$.

Proposition 5.10. If $\xi_{f}$ and $\xi_{g} \in \hat{\mathfrak{a}}$, then $\left[\xi_{f}, \xi_{g}\right](y)=0$ for all $y \in \hat{\mathfrak{g}}_{\mathcal{D}}$.

Proof. To prove the Proposition, it suffices to show that the flows corresponding to generating vector fields in $\hat{\mathfrak{a}}$ commute, i.e., for $i \leq k$,

$$
\begin{aligned}
\theta_{i, j}\left(\theta_{k, l}(y, s), t\right) & =\theta_{k, l}\left(\theta_{i, j}(y, t), s\right), \forall y \in \hat{\mathfrak{g}}_{\mathcal{D}} \\
\mu_{i, j}\left(\mu_{k, l}(y, s), t\right) & =\mu_{k, l}\left(\mu_{i, j}(y, t), s\right), \forall y \in \hat{\mathfrak{g}}_{\mathcal{D}} \\
\theta_{i, j}\left(\mu_{k, l}(y, s), t\right) & =\mu_{k, l}\left(\theta_{i, j}(y, t), s\right), \forall y \in \hat{\mathfrak{g}}_{\mathcal{D}}
\end{aligned}
$$

For this, let $u \in G_{i}$ and $v \in G_{k}$. For a point $q=\left(g_{1}, y^{1}, \ldots, g_{n}, y^{n}\right) \in \Pi_{a=1}^{n} G_{a} \times \mathfrak{g}_{a}$, define $h(q)=g_{i} u g_{i}^{-1}$ and $l(q)=g_{k} v g_{k}^{-1}$. Set

$$
\begin{gathered}
r_{i}(q)=\left(g_{1}, y^{1}, \ldots, g_{i}, y^{i}, h(q) g_{i+1}, y^{i+1}, \ldots, h(q) g_{n}, y^{n}\right), \\
w_{k}(q)=\left(g_{1}, y^{1}, \ldots, g_{k}, y^{k}, l(q) g_{k+1}, y^{k+1}, \ldots, l(q) g_{n}, y^{n}\right) .
\end{gathered}
$$

The identities in Equation (5.24) reduce to the equation

$$
r_{i}\left(w_{k}(q)\right)=w_{k}\left(r_{i}(q)\right)
$$

for particular choices of $u$ and $v$.

We first assume $i<k$. Then if $a \leq k$, it is routine to check that the $G_{a} \times \mathfrak{g}_{a}$ coordinates in Equation (5.25) coincide. For $a>k$, the $G_{a} \times \mathfrak{g}_{a}$-coordinate of $r_{i}\left(w_{k}(q)\right)$ is $\left(h(q) l(q) g_{a}, y^{a}\right)$, while the $G_{a} \times \mathfrak{g}_{a}$-coordinate of $w_{k}\left(r_{i}(q)\right)$ is $\left(h(q) l(q) h(q)^{-1} h(q) g_{a}, y^{a}\right)$, so Equation (5.25) is easily verified.

In the case $i=k$, note that Equation (5.25) is easy to verify when $u v=v u$. Thus, to verify Equations (5.24), it suffices to check that $u v=v u$ when $u$ and $v$ are chosen from $\exp \left(-t z_{i, j}\right)$ and $\exp \left(-s \nabla \phi_{i, l}\left(n^{i}\right)\right)$, for $j=1, \ldots, r_{i}$ and $l=1, \ldots, s_{i}$, and for any $t, s \in \mathbb{C}$. This follows by Lemma 5.5 .

Q.E.D.

We now show that $\hat{\mathfrak{a}}$ lifts the action of $\mathfrak{a}$ to $\hat{\mathfrak{g}}_{\mathcal{D}}$. 
Lemma 5.11. Let $\mu: \hat{\mathfrak{g}}_{\mathcal{D}} \rightarrow X_{\mathcal{D}}$ be the étale covering defined in Section 3.3. Then $\mu_{*} \hat{\mathfrak{a}}=\mathfrak{a}$, so that $\hat{\mathfrak{a}}$ lifts the action of $\mathfrak{a}$ to $\hat{\mathfrak{g}}_{\mathcal{D}}$.

Proof. Consider the commutative diagram

$$
\begin{array}{llclc}
\hat{\mathfrak{g}}_{\mathcal{D}} & \rightarrow & \dot{\mathfrak{g}}_{\mathcal{D}} & \stackrel{\alpha}{\rightarrow} & \tilde{\mathfrak{g}}_{\mathcal{D}} \\
\downarrow \mu & & \downarrow \mu & & \downarrow \Psi \\
X_{\mathcal{D}} & \stackrel{\gamma}{\rightarrow} & \prod_{i=1}^{n} D_{i} & \rightarrow & \prod_{i=1}^{n} D_{i}
\end{array}
$$

where the first square is Diagram (3.3) and the map $\Psi: \tilde{\mathfrak{g}}_{\mathcal{D}} \rightarrow \prod_{i=1}^{n} D_{i}$ is projection on $\prod_{i=1}^{n} D_{i}$. For $z \in \prod_{i=1}^{n} D_{i}$ we identify $T_{z}\left(\prod_{i=1}^{n} D_{i}\right)$ as a subspace of $T_{z}\left(\prod_{i=1}^{n} \mathfrak{g}_{i}\right)=\bigoplus_{i=1}^{n} \mathfrak{g}_{i}$. Let $y=\left(g_{1}, y^{1}, \ldots, g_{n}, y^{n}\right) \in \hat{\mathfrak{g}}_{\mathcal{D}}$. Then $\alpha(y)=\left(g_{1} P_{1}, x^{1}, \ldots, g_{n} P_{n}, x^{n}\right)$, where $x^{i}=\operatorname{Ad}\left(g_{i}\right) y^{i}$. We let $x=x^{n}$, so $x^{i}=x_{i}$ by Remark 3.11, By Propositions 5.3 and 5.8,

$$
\Psi_{\alpha(y), *} \alpha_{y, *} \xi_{q_{i, j}}=(0,0, \ldots, \underbrace{0}_{\mathrm{ith}}, \underbrace{\left[x_{i+1}, \operatorname{Ad}\left(g_{i}\right) z_{i, j}\right]}_{\mathrm{i}+1 \mathrm{st}}, \ldots, \underbrace{\left[x, \operatorname{Ad}\left(g_{i}\right) z_{i, j}\right]}_{\text {nth }}),
$$

for $1 \leq i \leq n-1,1 \leq j \leq r_{i}$, and

$$
\Psi_{\alpha(y), *} \alpha_{y, *} \xi_{p_{i, j}}=(0,0, \ldots, \underbrace{0}_{\text {ith }}, \underbrace{\left[x_{i+1}, \operatorname{Ad}\left(g_{i}\right) \nabla \phi_{i, j}\left(n^{i}\right)\right]}_{\mathrm{i}+1 \mathrm{st}}, \ldots, \underbrace{\left[x, \operatorname{Ad}\left(g_{i}\right) \nabla \phi_{i, j}\left(n^{i}\right)\right]}_{\text {nth }}),
$$

for $1 \leq i \leq n-1,1 \leq j \leq s_{i}$.

Recall that $\xi_{f_{i, j}} \in \mathfrak{a}$, where $f_{i, j}(x)=\operatorname{tr}\left(x_{i}^{j}\right)$. Then identifying $T_{z}(\mathfrak{g})=\mathfrak{g}$ for $z \in \mathfrak{g}$, it follows from Theorem 2.12 in [KW06a] that $\left(\xi_{f_{i, j}}\right)_{z}=\left[j z_{i}^{j-1}, z\right]$. Hence,

$$
\gamma_{x, *} \xi_{f_{i, j}}=\left(\left[j x_{i}^{j-1}, x\right]_{1},\left[j x_{i}^{j-1}, x\right]_{2}, \ldots,\left[j x_{i}^{j-1}, x\right]\right) .
$$

From Remark [5.1, it follows that for $k>i,\left[j x_{i}^{j-1}, x\right]_{k}=\left[j x_{i}^{j-1}, x_{k}\right]$ and for $k \leq i$, $\left[j x_{i}^{j-1}, x\right]_{k}=0$. Thus,

$$
\gamma_{x, *} \xi_{f_{i, j}}=(0,0 \ldots, \underbrace{0}_{\mathrm{ith}}, \underbrace{\left[j x_{i}^{j-1}, x_{i+1}\right]}_{\mathrm{i}+1 \mathrm{st}}, \ldots,\left[j x_{i}^{j-1}, x\right])
$$

Since $y \in \hat{\mathfrak{g}}_{\mathcal{D}}, x \in \mathfrak{g}_{\text {sreg }}$ by Remark 3.11, so that by Theorem 2.1, $x_{i}$ is regular for all $i$. Thus,

$$
\operatorname{span}\left\{x_{i}^{j-1}: 1 \leq j \leq i\right\}=\mathfrak{z} \mathfrak{g}_{i}\left(x_{i}\right),
$$

by a standard result from linear algebra. We claim

$$
\operatorname{span}\left\{\operatorname{Ad}\left(g_{i}\right) z_{i, j}, \operatorname{Ad}\left(g_{i}\right) \nabla \phi_{i, k}\left(n^{i}\right): 1 \leq j \leq r_{i}, 1 \leq k \leq s_{i}\right\}=\mathfrak{z}_{\mathfrak{g}_{i}}\left(x_{i}\right)
$$


Indeed, $\operatorname{Ad}\left(g_{i}^{-1}\right) x_{i}=y^{i}=s^{i}+n^{i}$ with $n^{i} \in \mathfrak{s}_{i}$ principal nilpotent. By Remark 5.7, $\left\{\nabla \phi_{i, k}\left(n^{i}\right): 1 \leq k \leq s_{i}\right\}$ is a basis of $\mathfrak{z}_{\mathfrak{s}_{i}}\left(n^{i}\right)$. Since $\mathfrak{z}_{\mathfrak{g}_{i}}\left(y^{i}\right)=\mathfrak{z}_{i} \oplus \mathfrak{z}_{\mathfrak{s}_{i}}\left(n^{i}\right)$, the claim follows easily.

By Equations (5.27), (5.28), and (5.29), $\operatorname{span}\left\{\gamma_{x, *} \xi_{f_{i, j}}: 1 \leq j \leq i-1\right\}$ equals

$$
\operatorname{span}\left\{\Psi_{\alpha(y), *} \alpha_{y, *} \xi_{q_{i, j}}: 1 \leq j \leq r_{i}\right\} \oplus \operatorname{span}\left\{\Psi_{\alpha(y), *} \alpha_{y, *} \xi_{p_{i, k}}: 1 \leq k \leq s_{i}\right\}
$$

for each $i, 1 \leq i \leq n-1$ and any $y \in \hat{\mathfrak{g}}_{\mathcal{D}}$. Thus, $\gamma_{*} \mathfrak{a}=\Psi_{*} \alpha_{*} \hat{\mathfrak{a}}=\gamma_{*} \mu_{*} \hat{\mathfrak{a}}$, so since $\gamma$ is an embedding, $\mathfrak{a}=\mu_{*} \hat{\mathfrak{a}}$.

\section{Q.E.D.}

5.4. The integration of the $\hat{\mathfrak{a}}$-action on $\hat{\mathfrak{g}}_{\mathcal{D}}$. In this section, we show that the Lie algebra $\hat{\mathfrak{a}}$ integrates to an algebraic action of a connected abelian algebraic group on $\hat{\mathfrak{g}}_{\mathcal{D}}$.

As before, $\mathcal{D}=\left(D_{1}, \ldots, D_{n}\right)$ is regular decomposition data with $D_{i}=G_{i} \cdot\left(\mathfrak{z}_{i, g e n}+u_{i}\right)$. Recall the identification from Section 3.3 ,

$$
\hat{\mathfrak{g}}_{\mathcal{D}} \cong \mathcal{D}_{c}=\left\{\left(x, z_{1}, \ldots, z_{n}\right) \in \mathfrak{g}_{\text {sreg }} \times \mathfrak{z}_{\mathcal{D}}: x_{i} \in G_{i} \cdot\left(z_{i}+u_{i}\right)\right\}
$$

and the projections $\mu: \mathcal{D}_{c} \rightarrow \mathfrak{g}_{\text {sreg }}$ and $\kappa: \mathcal{D}_{c} \rightarrow \mathfrak{z}_{\mathcal{D}}$. For $\left(z_{1}, \ldots, z_{n}\right) \in \mathfrak{z}_{\mathcal{D}}$,

$\kappa^{-1}\left(z_{1}, \ldots, z_{n}\right) \stackrel{\mu}{\cong}\left\{x \in X_{\mathcal{D}}: x_{i} \in G_{i} \cdot\left(z_{i}+u_{i}\right), i=1, \ldots, n\right\}=\Phi^{-1}\left(\Phi_{1}\left(z_{1}\right), \ldots, \Phi_{n}\left(z_{n}\right)\right) \cap \mathfrak{g}_{\text {sreg }}$ by Lemma 3.6 .

It is convenient to normalize the nilpotent matrix $u_{i}$ so that $u_{i}=e^{i}$ is the unique principal nilpotent element of $\mathfrak{l}_{i}$ in Jordan canonical form. Let $Z_{D_{i}}=Z_{G_{i}}\left(\mathfrak{z}_{i}+e^{i}\right)=Z_{L_{i}}\left(e_{i}\right)$. Note that $Z_{D_{i}}$ is connected and abelian because it is the centralizer of a regular element of $\mathfrak{l}_{i}$ (see Proposition 14 in [Kos63]). We identify

$$
\dot{D}\left(\mathfrak{l}_{i}, e^{i}\right)=G_{i} \times_{L_{i}}\left(\mathfrak{z}_{i, g e n}+L_{i} \cdot e^{i}\right) \cong G_{i} \times_{Z_{D_{i}}}\left(\mathfrak{z}_{i, g e n}+e^{i}\right),
$$

and identify $\dot{\mathfrak{g}}_{\mathcal{D}} \cong \Pi_{i=1}^{n} G_{i} \times_{Z_{D_{i}}}\left(\mathfrak{z}_{i, g e n}+e^{i}\right)$. The abelian algebraic group $Z_{D_{i}}$ has Levi decomposition $Z_{D_{i}}=Z_{i} \times\left(Z_{D_{i}}\right)_{u}$ with unipotent radical $\left(Z_{D_{i}}\right)_{u}$, and its Lie algebra $\mathfrak{z}_{D_{i}}=\mathfrak{z}_{i} \oplus \mathfrak{z}_{\mathfrak{s}_{i}}\left(e^{i}\right)$. Recall that $\exp : \mathfrak{z}_{\mathfrak{s}_{i}}\left(e^{i}\right) \rightarrow\left(Z_{D_{i}}\right)_{u}$ is an isomorphism. Further $\mathfrak{z}_{\mathfrak{s}_{i}}\left(e^{i}\right)$ has basis given by the elements $\nabla \phi_{i, j}\left(e^{i}\right)$, for $j=1, \ldots, s_{i}$ by Remark 5.7 .

Lemma 5.12. If $x \in \mathfrak{g}_{\text {sreg }}$, then $Z_{G_{i}}\left(x_{i}\right) \cap Z_{G_{i+1}}\left(x_{i+1}\right)=\{e\}$ for all $1 \leq i \leq n-1$, where $e \in G_{n}$ denotes the identity matrix.

Proof. If $A \in Z_{G_{i}}\left(x_{i}\right) \cap Z_{G_{i+1}}\left(x_{i+1}\right)$, then it follows easily that $A-e \in \mathfrak{z}_{\mathfrak{g}_{i}}\left(x_{i}\right) \cap \mathfrak{z} \mathfrak{g}_{i+1}\left(x_{i+1}\right)=$ 0 by Theorem 2.1, which implies the lemma.

\section{Q.E.D.}

We consider the connected, abelian algebraic group $Z_{\mathcal{D}}=Z_{D_{1}} \times \cdots \times Z_{D_{n-1}}$. 
Theorem 5.13. (1) The Lie algebra $\hat{\mathfrak{a}}$ integrates to a free algebraic action of the group $Z_{\mathcal{D}}$ on $\hat{\mathfrak{g}}_{\mathcal{D}}$. This action of $Z_{\mathcal{D}}$ preserves the fibers $\kappa^{-1}\left(z_{1}, \ldots, z_{n}\right)$ for $\left(z_{1}, \ldots, z_{n}\right) \in \mathfrak{z}_{\mathcal{D}}$.

(2) The orbits of $Z_{\mathcal{D}}$ in $\kappa^{-1}\left(z_{1}, \ldots, z_{n}\right)$ are the irreducible components of $\kappa^{-1}\left(z_{1}, \ldots, z_{n}\right)$. If we let $j_{i}$ denote the cardinality of the set $\sigma_{i}\left(z_{i}\right) \cap \sigma_{i+1}\left(z_{i+1}\right)$ for $1 \leq i \leq n-1$, then the number of $Z_{\mathcal{D}}$-orbits in $\kappa^{-1}\left(z_{1}, \ldots, z_{n}\right)$ is exactly $2^{\sum_{i=1}^{n-1} j_{i}}$.

Proof. Let $k_{i} \in Z_{D_{i}}$ and let $y=\left(g_{1}, y^{1}, \ldots, g_{n}, y^{n}\right) \in \hat{\mathfrak{g}}_{\mathcal{D}}$ with $y^{i} \in \mathfrak{z}_{i, \text { gen }}+e^{i}$. The group $Z_{D_{i}}$ acts on $\hat{\mathfrak{g}}_{\mathcal{D}}$ by the formula

$$
k_{i} \cdot y=\left(g_{1}, y^{1}, \ldots, g_{i}, y^{i}, g_{i} k_{i} g_{i}^{-1} g_{i+1}, y^{i+1}, g_{i} k_{i} g_{i}^{-1} g_{n}, y^{n}\right) .
$$

We claim that $T\left(Z_{D_{i}} \cdot y\right)=\hat{\mathfrak{a}}_{i}($ see (15.23) $)$. Indeed, $T_{y}\left(Z_{D_{i}} \cdot y\right)=T_{y}\left(Z_{i} \cdot y\right)+T_{y}\left(\left(Z_{D_{i}}\right)_{u} \cdot y\right)$. By Equations (5.8) and (5.22), for $y \in \hat{\mathfrak{g}}_{\mathcal{D}}, T_{y}\left(Z_{i} \cdot y\right)=\operatorname{span}\left\{\xi_{q_{i, j}}(y): j=1, \ldots, r_{i}\right\}$ and $T_{y}\left(\left(Z_{D_{i}}\right)_{u} \cdot y\right)=\operatorname{span}\left\{\xi_{p_{i, k}}(y): k=1, \ldots, s_{i}\right\}$, which gives the claim. Hence, $\hat{\mathfrak{a}}_{i}$ induces the tangent space to the $Z_{D_{i}}$-action at every point $y$ of $\hat{\mathfrak{g}}_{\mathcal{D}}$, so the action of $\hat{\mathfrak{a}}_{i}$ integrates to the algebraic action of the algebraic group $Z_{D_{i}}$ on $\hat{\mathfrak{g}}_{\mathcal{D}}$.

Using Equation (5.24), it is easy to verify that the actions of $Z_{D_{i}}$ and $Z_{D_{k}}$ commute for $i \neq k$. Hence, $Z_{\mathcal{D}}$ acts on $\hat{\mathfrak{g}}_{\mathcal{D}}$ by the formula

$$
\left(k_{1}, \ldots, k_{n-1}\right) \cdot y=k_{1} \cdots k_{n-1} \cdot y, k_{i} \in Z_{D_{i}}, y \in \hat{\mathfrak{g}}_{\mathcal{D}}
$$

or more explicitly,

$$
\left(k_{1}, \ldots, k_{n-1}\right) \cdot y=
$$

$$
(g_{1}, y^{1}, g_{1} k_{1} g_{1}^{-1} g_{2}, y^{2}, \ldots, \underbrace{g_{1} k_{1} g_{1}^{-1} \ldots g_{i-1} k_{i-1} g_{i-1}^{-1} g_{i}, y^{i}}_{\text {ith }}, \ldots, \underbrace{g_{1} k_{1} g_{1}^{-1} \ldots g_{n-1} k_{n-1} g_{n-1}^{-1} g_{n}, y^{n}}_{\text {nth }}) .
$$

Since $\hat{\mathfrak{a}}_{i}$ integrates to an algebraic action of $Z_{D_{i}}$ for each $i, 1 \leq i \leq n-1$, and the actions of the groups $Z_{D_{i}}$ commute, it follows that $\hat{\mathfrak{a}}$ integrates to an algebraic action of $Z_{\mathcal{D}}$ on $\hat{\mathfrak{g}}_{\mathcal{D}}$. By Equations (5.32) and (5.33), the $Z_{\mathcal{D}}$-action on $\hat{\mathfrak{g}}_{\mathcal{D}}$ preserves the fibers $\kappa^{-1}\left(z_{1}, \ldots, z_{n}\right)$ for $\left(z_{1}, \ldots, z_{n}\right) \in \mathfrak{z}_{\mathcal{D}}$.

To prove that the $Z_{\mathcal{D}}$-action on $\hat{\mathfrak{g}}_{\mathcal{D}}$ is free, we show by induction that if $k=\left(k_{1}, \ldots, k_{n-1}\right)$ fixes $y \in \hat{\mathfrak{g}}_{\mathcal{D}}$, and $k_{1}, \ldots, k_{i-1}=e$, then $k_{i}=e$. Indeed, then $k \cdot y$ has $G_{i+1} \times \mathfrak{g}_{i+1}$ coordinate $\left(g_{i} k_{i} g_{i}^{-1} g_{i+1}, y^{i+1}\right)$, so by hypothesis, $\operatorname{Ad}\left(g_{i} k_{i} g_{i}^{-1} g_{i+1}\right) y^{i+1}=\operatorname{Ad}\left(g_{i+1}\right) y^{i+1}$. Thus, if we set $x^{i}=\operatorname{Ad}\left(g_{i}\right) y^{i}$ and $x^{i+1}=\operatorname{Ad}\left(g_{i+1}\right) y^{i+1}$, then $g_{i} k_{i} g_{i}^{-1} \in Z_{G_{i}}\left(x^{i}\right) \cap Z_{G_{i+1}}\left(x^{i+1}\right)=\{e\}$ by Lemma 5.12. Hence, $k_{i}=e$, which establishes the inductive step, so the $Z_{\mathcal{D}^{-}}$action is free.

To prove (2), we first observe that since the connected algebraic group $Z_{\mathcal{D}}$ acts freely on $\hat{\mathfrak{g}}_{\mathcal{D}}$, each $Z_{\mathcal{D}^{-}}$orbit in $\kappa^{-1}\left(z_{1}, \ldots, z_{n}\right)$ is an irreducible subvariety of $\kappa^{-1}\left(z_{1}, \ldots, z_{n}\right)$ of dimension $\left(\begin{array}{l}n \\ 2\end{array}\right)$. It follows that all orbits of $Z_{\mathcal{D}}$ on $\kappa^{-1}\left(z_{1}, \ldots, z_{n}\right)$ are closed. Let 
$c=\left(\Phi_{1}\left(z_{1}\right), \ldots, \Phi_{n}\left(z_{n}\right)\right)$. Using the isomorphism $\mu: \kappa^{-1}\left(z_{1}, \ldots, z_{n}\right) \rightarrow \Phi^{-1}(c)_{\text {sreg }}$ of Equation (5.31), it follows that if $x \in \kappa^{-1}\left(z_{1}, \ldots, z_{n}\right)$, then $\mu\left(Z_{\mathcal{D}} \cdot x\right)$ is a closed, irreducible subvariety of dimension $\left(\begin{array}{l}n \\ 2\end{array}\right)$ of $\Phi^{-1}(c)_{\text {sreg. }}$. By Theorem 3.12 in [KW06a], each irreducible component of $\Phi^{-1}(c)_{\text {sreg }}$ is an $A$-orbit of dimension $\left(\begin{array}{l}n \\ 2\end{array}\right)$, which implies the first statement of (2). The last statement of (2) now follows from Theorem 2.3.

Q.E.D.

Remark 5.14. Let $\mathcal{D}=\left(D_{1}, \ldots, D_{n}\right)$ with $D_{i}=D\left(\mathfrak{l}_{i}, u_{i}\right)$ and suppose for each $i$ that all blocks of $\mathfrak{l}_{i}$ have different sizes (see Remarks [3.5, 3.13). In this case, Theorem 5.13 implies that $\mathfrak{a}$ integrates to an algebraic action of $Z_{\mathcal{D}}$ on $X_{\mathcal{D}}$.

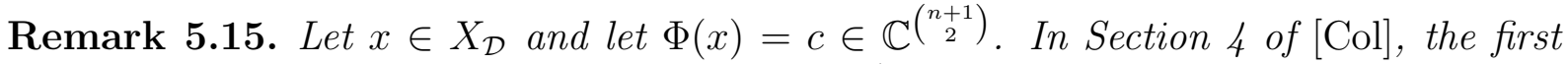
author constructed an algebraic $Z_{\mathcal{D}}$-action on $\Phi^{-1}(c)_{\text {sreg }}$ (see Theorem [2.3). For $y \in \hat{\mathfrak{g}}_{\mathcal{D}}$ such that $\mu(y) \in \Phi^{-1}(c)_{\text {sreg }}$ and $z \in Z_{\mathcal{D}}, \mu(z \cdot y)=z \cdot \mu(y)$. This can be verified using the formula for the action in [Col]. However, the $Z_{\mathcal{D}}$-action on each fiber $\Phi^{-1}(c)_{\text {sreg }}$ does not in general extend to a global algebraic $Z_{\mathcal{D}}$-action on $X_{\mathcal{D}}$ that integrates the a-action, but the lift $\hat{\mathfrak{a}}$ of $\mathfrak{a}$ does integrate to a global algebraic action on the covering $\hat{\mathfrak{g}}_{\mathcal{D}}$ by the previous theorem. In addition, we regard the formula for the $Z_{\mathcal{D}}$-action given in Equation (5.33) as much simpler than the formula given from [Col].

5.5. Generic elements and irreducibility of $\hat{\mathfrak{g}}_{\mathcal{D}}$. In this section, we show that $\hat{\mathfrak{g}}_{\mathcal{D}}$ and $X_{\mathcal{D}}$ are connected by considering the open subset

$$
\mathfrak{z}_{\mathcal{D}, \text { gen }}:=\left\{\left(z_{1}, \ldots, z_{n}\right) \in \mathfrak{z}_{\mathcal{D}}: \sigma_{i}\left(z_{i}\right) \cap \sigma_{i+1}\left(z_{i+1}\right)=\emptyset, i=1, \ldots, n-1\right\},
$$

its preimage $\hat{\mathfrak{g}}_{\mathcal{D} \text {,gen }}:=\kappa^{-1}\left(\mathfrak{z}_{\mathcal{D}, \text { gen }}\right)$, and $X_{\mathcal{D} \text {, gen }}:=\mu\left(\hat{\mathfrak{g}}_{\mathcal{D}, \text { gen }}\right)$. We further show that when each $D_{i}$ consists of regular semisimple elements, then $\mu: \hat{\mathfrak{g}}_{\mathcal{D} \text {,gen }} \rightarrow X_{\mathcal{D} \text {, gen }}$ specializes to a covering considered by Kostant and Wallach in [KW06b], and generalize a result in [KW06b] concerning Hessenberg matrices to our setting.

Note that

$$
\begin{array}{r}
\hat{\mathfrak{g}}_{\mathcal{D}, \text { gen }}=\left\{\left(x, z_{1}, \ldots, z_{n}\right): x \in X_{\mathcal{D}, \text { gen }},\left(z_{1}, \ldots, z_{n}\right) \in \mathfrak{z}_{\mathcal{D}, \text { gen }}\right\}, \\
X_{\mathcal{D}, \text { gen }}=\left\{x \in X_{\mathcal{D}}: \sigma_{i}\left(x_{i}\right) \cap \sigma_{i+1}\left(x_{i+1}\right)=\emptyset\right\} .
\end{array}
$$

It follows from definitions that $Z_{\mathcal{D}}$ acts on $\hat{\mathfrak{g}}_{\mathcal{D}, g e n}$.

Corollary 5.16. For $\left(z_{1}, \ldots, z_{n}\right) \in \mathfrak{z}_{\mathcal{D} \text {,gen }}$, the group $Z_{\mathcal{D}}$ acts simply transitively on the fibers $\kappa^{-1}\left(z_{1}, \ldots, z_{n}\right)$.

Proof. Since the cardinality of the sets $\sigma_{i}\left(z_{i}\right) \cap \sigma_{i+1}\left(z_{i+1}\right)$ is zero for $i=1, \ldots, n-1$, the corollary follows by Theorem 5.13 . 
Proposition 5.17. The map $\mu: \hat{\mathfrak{g}}_{\mathcal{D}, \text { gen }} \rightarrow X_{\mathcal{D} \text {,gen }}$ is a $\Sigma_{\mathcal{D}^{-}}$covering and $\hat{\mathfrak{g}}_{\mathcal{D}, \text { gen }}$ is smooth and irreducible.

Proof. By Theorem 3.12, $\mu: \hat{\mathfrak{g}}_{\mathcal{D} \text {,gen }} \rightarrow X_{\mathcal{D} \text {,gen }}$ is a $\Sigma_{\mathcal{D}}$-covering of smooth varities, so it suffices to show that $\hat{\mathfrak{g}}_{\mathcal{D} \text {,gen }}$ is connected. By Remark $3.14, \kappa: \hat{\mathfrak{g}}_{\mathcal{D} \text {, gen }} \rightarrow \mathfrak{z}_{\mathcal{D} \text {, gen }}$ is a surjective submersion, so $\kappa$ is smooth of relative dimension $n^{2}-\left(\begin{array}{c}n+1 \\ 2\end{array}\right)$ ([Har77], Proposition III.10.4) and hence flat ([Har77], Theorem III.10.2). Hence by Exercise III.9.1 in [Har77], it follows that $\kappa$ is an open morphism. By Corollary 5.16, $\kappa$ has connected fibers, and it is clear that $\mathfrak{z}_{\mathcal{D} \text {,gen }}$ is connected. The Proposition now follows from the following easy fact: if $f: X \rightarrow Y$ is a surjective, open morphism, and $Y$ and all fibers of $f$ are connected, then $X$ is connected.

\section{Q.E.D.}

Theorem 5.18. The varieties $\hat{\mathfrak{g}}_{\mathcal{D}}$ and $X_{\mathcal{D}}$ are connected and irreducible.

Proof. By Theorems 3.10 and 3.12, and the fact that $\mu: \hat{\mathfrak{g}}_{\mathcal{D}} \rightarrow X_{\mathcal{D}}$ is surjective, it suffices to prove that $\hat{\mathfrak{g}}_{\mathcal{D}}$ is connected. Let $\hat{\mathfrak{g}}_{\mathcal{D}}=Y_{1} \cup \cdots \cup Y_{k}$ be the connected components of $\hat{\mathfrak{g}}_{\mathcal{D}}$. By Proposition 5.17, we may assume that $\hat{\mathfrak{g}}_{\mathcal{D}, \text { gen }} \subset Y_{1}$. Hence, if $i>1, Y_{i} \subset$ $\hat{\mathfrak{g}}_{\mathcal{D}, s}:=\hat{\mathfrak{g}}_{\mathcal{D}}-\hat{\mathfrak{g}}_{\mathcal{D}, \text { gen }}$. By the dimension assertion in Theorem $[3.12$, it suffices to prove that $\operatorname{dim}\left(\hat{\mathfrak{g}}_{\mathcal{D}, s}\right)<\operatorname{dim}\left(\hat{\mathfrak{g}}_{\mathcal{D}}\right)$.

For this, since $\kappa: \hat{\mathfrak{g}}_{\mathcal{D}} \rightarrow \mathfrak{z}_{\mathcal{D}}$ is smooth of relative dimension $n^{2}-\left(\begin{array}{c}n+1 \\ 2\end{array}\right)$, for any locally closed subvariety $Y \subset \mathfrak{z}_{\mathcal{D}}, \operatorname{dim}\left(\kappa^{-1}(Y)\right)=\operatorname{dim}(Y)+n^{2}-\left(\begin{array}{c}n+1 \\ 2\end{array}\right)$ by Proposition III.10.1(b) of [Har77]. Let $\mathfrak{z}_{\mathcal{D}, s}:=\mathfrak{z}_{\mathcal{D}}-\mathfrak{z}_{\mathcal{D} \text {,gen }}$, and note that $\hat{\mathfrak{g}}_{\mathcal{D}, s}=\kappa^{-1}\left(\mathfrak{z}_{\mathcal{D}, s}\right)$. It follows easily that $\operatorname{dim}\left(\hat{\mathfrak{g}}_{\mathcal{D}, s}\right)=\operatorname{dim}\left(\mathfrak{z}_{\mathcal{D}, s}\right)+n^{2}-\left(\begin{array}{c}n+1 \\ 2\end{array}\right)$ and $\operatorname{dim}\left(\hat{\mathfrak{g}}_{\mathcal{D}}\right)=\operatorname{dim}\left(\mathfrak{z}_{\mathcal{D}}\right)+n^{2}-\left(\begin{array}{c}n+1 \\ 2\end{array}\right)$. At the beginning of Section 5.1, we identify $\mathbb{C}^{r_{i}} \cong \mathfrak{z}_{i}$, and we can then identify the variety $\mathfrak{z}_{\mathcal{D}}$ with an open subset in $\mathbb{C}^{r} \cong \sum_{i=1}^{n} \mathfrak{z}_{i}, r=\sum_{i=1}^{n} r_{i}$. Using this identification, $\mathfrak{z}_{\mathcal{D}, s}$ is identified with an open subset of a union of hyperplanes in $\mathbb{C}^{r}$. It follows that $\operatorname{dim}\left(\mathfrak{z}_{\mathcal{D}, s}\right)<\operatorname{dim}\left(\mathfrak{z}_{\mathcal{D}}\right)$, so $\operatorname{dim}\left(\hat{\mathfrak{g}}_{\mathcal{D}, s}\right)<\operatorname{dim}\left(\hat{\mathfrak{g}}_{\mathcal{D}}\right)$

\section{Q.E.D.}

Remark 5.19. Corollary 5.16 and Proposition 5.17 specialize to give results proved by Kostant and Wallach in [KW06b]. Indeed, suppose that each $D_{i}$ consists of regular semisimple elements. Then Proposition 5.17 implies that

$$
\hat{\mathfrak{g}}_{\mathcal{D}, \text { gen }} \cong M_{\Omega}(n, \mathfrak{e}):=\left\{\left(x, z_{1}, \ldots, z_{n}\right):\left(z_{1}, \ldots, z_{n}\right) \in \mathfrak{z}_{\mathcal{D}, g e n}, x_{i} \in G_{i} \cdot z_{i}, i=1, \ldots, n\right\}
$$

is a covering of $X_{\mathcal{D} \text {,gen }}$ by a product of symmetric groups, which is Theorem 4.14 in KW06b]. Further, in this case $Z_{\mathcal{D}} \cong\left(\mathbb{C}^{\times}\right)^{\left(\begin{array}{c}n \\ 2\end{array}\right)}$, and the algebraic action of $Z_{\mathcal{D}}$ on $\hat{\mathfrak{g}}_{\mathcal{D} \text {,gen }}$ is easily seen to coincide with the algebraic action of $\left(\mathbb{C}^{\times}\right)^{\left(\begin{array}{c}n \\ 2\end{array}\right)}$ in [KW06b] which lifts the a-action on $M_{\Omega}(n)$.

We use Corollary 5.16 to obtain an analogue of Theorem 5.12 in KW06b. In Section 2. we introduced the variety of upper Hessenberg matrices $\mathfrak{b}+e$ and noted that the 
Kostant-Wallach map restricts to an isomorphism of varieties $\Phi: \mathfrak{b}+e \rightarrow \mathbb{C}^{\left(\begin{array}{c}n+1 \\ 2\end{array}\right)}$ and that $\mathfrak{b}+e \subset \mathfrak{g}_{\text {sreg }}$. We define a closed subvariety of $\hat{\mathfrak{g}}_{\mathcal{D}, \text { gen }}$ :

$$
\left(\hat{\mathfrak{g}}_{\mathcal{D}, \text { gen }}\right)_{\mathfrak{b}+e}=\left\{\left(g_{1}, y^{1}, \ldots, g_{n}, y^{n}\right) \in \hat{\mathfrak{g}}_{\mathcal{D}, \text { gen }}: \operatorname{Ad}\left(g_{n}\right) y^{n} \in \mathfrak{b}+e\right\}
$$

Theorem 5.20. The morphism $\phi: Z_{\mathcal{D}} \times\left(\hat{\mathfrak{g}}_{\mathcal{D}, \text { gen }}\right)_{\mathfrak{b}+e} \rightarrow \hat{\mathfrak{g}}_{\mathcal{D} \text {,gen }}$ given by $(k, x) \rightarrow k \cdot x$, $k \in Z_{\mathcal{D}}, x \in\left(\hat{\mathfrak{g}}_{\mathcal{D}, \text { gen }}\right)_{\mathfrak{b}+e}$ is an isomorphism of algebraic varieties. Thus, $\hat{\mathfrak{g}}_{\mathcal{D} \text {,gen }}$ is a Zariski trivial $Z_{\mathcal{D}}$-principal bundle over $\left(\hat{\mathfrak{g}}_{\mathcal{D}, \text { gen }}\right)_{\mathfrak{b}+e}$.

Proof. By Proposition 5.17] and Zariski's main theorem (see [TY05] Corollary 17.4.8), it suffices to show that $\phi$ is bijective. By Theorem 5.13 (1), the morphism $\phi$ is injective. Let $\left(x, z_{1}, \ldots, z_{n}\right) \in \hat{\mathfrak{g}}_{\mathcal{D}, \text { gen }}$, so $\left(z_{1}, \ldots, z_{n}\right) \in \mathfrak{z}_{\mathcal{D}, \text { gen }}$, and let $c=\Phi(x)$. There is a unique $\tilde{x} \in \Phi^{-1}(c)_{\text {sreg }} \cap(\mathfrak{b}+e)$. Since $\mu: \kappa^{-1}\left(z_{1}, \ldots, z_{n}\right) \rightarrow \Phi^{-1}(c)_{\text {sreg }}$ is an isomorphism by Equation (5.31), it follows that $\left(\tilde{x}, z_{1}, \ldots, z_{n}\right) \in\left(\hat{\mathfrak{g}}_{\mathcal{D}, g e n}\right)_{\mathfrak{b}+e}$. Since $x$ and $\tilde{x}$ are elements of $\kappa^{-1}\left(z_{1}, \ldots, z_{n}\right)$, by Corollary [5.16 there exists $k \in Z_{\mathcal{D}}$ such that $k \cdot \tilde{x}=x$, so $\phi$ is surjective.

\section{Q.E.D.}

Remark 5.21. By Theorem 5.20, the variety $\left(\hat{\mathfrak{g}}_{\mathcal{D}, \text { gen }}\right)_{\mathfrak{b}+e}$ is a smooth and irreducible closed subvariety of $\hat{\mathfrak{g}}_{\mathcal{D} \text {,gen }}$. Moreover, the projection $\kappa: \hat{\mathfrak{g}}_{\mathcal{D}, \text { gen }} \rightarrow \mathfrak{z}_{\mathcal{D} \text {,gen }}$ restricts to an isomorphism of varieties $\left(\hat{\mathfrak{g}}_{\mathcal{D}, \text { gen }}\right)_{\mathfrak{b}+e} \rightarrow \mathfrak{z}_{\mathcal{D}, \text { gen }}$. This last assertion can be proved using the argument from the proof of the last theorem.

\section{REFERENCES}

[BP08] Roger Bielawski and Victor Pidstrygach, Gelfand-Zeitlin actions and rational maps, Math. Z. 260 (2008), no. 4, 779-803.

[Bro98a] Abraham Broer, Decomposition varieties in semisimple Lie algebras, Canad. J. Math. 50 (1998), no. 5, 929-971.

[Bro98b] Abraham Broer, Lectures on decomposition classes, Representation theories and algebraic geometry (Montreal, PQ, 1997), NATO Adv. Sci. Inst. Ser. C Math. Phys. Sci., vol. 514, Kluwer Acad. Publ., Dordrecht, 1998, pp. 39-83.

[CG97] Neil Chriss and Victor Ginzburg, Representation theory and complex geometry, Birkhäuser Boston Inc., Boston, MA, 1997.

[Col] Mark Colarusso, The orbit structure of the gelfand-zeitlin group on $n \times n$ matrices, arXiv:0811.1351v1 [math.SG], to appear.

[Col07] Mark Colarusso, The gelfand-zeitlin algebra and polarizations of regular adjoint orbits for classical groups, Ph.D. thesis, University of California, San Diego, 2007.

[EL07] Sam Evens and Jiang-Hua Lu, Poisson geometry of the Grothendieck resolution of a complex semisimple group, Mosc. Math. J. 7 (2007), no. 4, 613-642.

[Har77] Robin Hartshorne, Algebraic geometry, Springer-Verlag, New York, 1977, Graduate Texts in Mathematics, No. 52.

[KKS78] David Kazhdan, Bertram Kostant, and Shlomo Sternberg, Hamiltonian group actions and dynamical systems of Calogero type, Comm. Pure Appl. Math. 31 (1978), no. 4, 481-507.

[Kos63] Bertram Kostant, Lie group representations on polynomial rings, Amer. J. Math. 85 (1963), $327-404$. 
[KW06a] Bertram Kostant and Nolan Wallach, Gelfand-Zeitlin theory from the perspective of classical mechanics. I, Studies in Lie theory, Progr. Math., vol. 243, Birkhäuser Boston, Boston, MA, 2006, pp. 319-364.

[KW06b] Bertram Kostant and Nolan Wallach, Gelfand-Zeitlin theory from the perspective of classical mechanics. II, The unity of mathematics, Progr. Math., vol. 244, Birkhäuser Boston, Boston, MA, 2006, pp. 387-420.

[TY05] Patrice Tauvel and Rupert W. T. Yu, Lie algebras and algebraic groups, Springer Monographs in Mathematics, Springer-Verlag, Berlin, 2005.

Department of Mathematics, University of Notre Dame, Notre Dame, 46556

E-mail address: mcolarus@nd.edu

Department of Mathematics, University of Notre Dame, Notre Dame, 46556

E-mail address: sevens@nd.edu 